\title{
Self-consistent Vlasov-Maxwell description of the longitudinal dynamics of intense charged particle beams
}

\author{
Ronald C. Davidson and Edward A. Startsev \\ Plasma Physics Laboratory, Princeton University, Princeton, New Jersey 08543, USA
}

(Received 22 December 2003; published 18 February 2004)

\begin{abstract}
This paper describes a self-consistent kinetic model for the longitudinal dynamics of a long, coasting beam propagating in straight (linear) geometry in the $z$ direction in the smooth-focusing approximation. Starting with the three-dimensional Vlasov-Maxwell equations, and integrating over the phasespace $\left(\mathbf{x}_{\perp}, \mathbf{p}_{\perp}\right)$ transverse to beam propagation, a closed system of equations is obtained for the nonlinear evolution of the longitudinal distribution function $F_{b}\left(z, p_{z}, t\right)$ and average axial electric field $\left\langle E_{z}^{s}\right\rangle(z, t)$. The primary assumptions in the present analysis are that the dependence on axial momentum $p_{z}$ of the distribution function $f_{b}(\mathbf{x}, \mathbf{p}, t)$ is factorable, and that the transverse beam dynamics remains relatively quiescent (absence of transverse instability or beam mismatch). The analysis is carried out correct to order $k_{z}^{2} r_{w}^{2}$ assuming slow axial spatial variations with $k_{z}^{2} r_{w}^{2} \ll 1$, where $k_{z} \sim \partial / \partial z$ is the inverse length scale of axial variation in the line density $\lambda_{b}(z, t)=\int d p_{z} F_{b}\left(z, p_{z}, t\right)$, and $r_{w}$ is the radius of the conducting wall (assumed perfectly conducting). A closed expression for the average longitudinal electric field $\left\langle E_{z}^{s}\right\rangle(z, t)$ in terms of geometric factors, the line density $\lambda_{b}$, and its derivatives $\partial \lambda_{b} / \partial z, \ldots$ is obtained for the class of bell-shaped density profiles $n_{b}(r, z, t)=\left(\lambda_{b} / \pi r_{b}^{2}\right) f\left(r / r_{b}\right)$, where the shape function $f\left(r / r_{b}\right)$ has the form specified by $f\left(r / r_{b}\right)=(n+1)\left(1-r^{2} / r_{b}^{2}\right)^{n}$ for $0 \leq r<r_{b}$, and $f\left(r / r_{b}\right)=$ 0 for $r_{b}<r \leq r_{w}$, where $n=0,1,2, \ldots$. The general kinetic formalism developed here is valid for the entire range of beam intensities (proportional to $\lambda_{b}$ ) ranging from low-intensity, emittance-dominated beams, to very-high-intensity, low-emittance beams.
\end{abstract}

DOI: 10.1103/PhysRevSTAB.7.024401

\section{INTRODUCTION}

High energy accelerators, transport systems, and storage rings [1-8] have a wide range of applications ranging from basic research in high energy and nuclear physics, to applications such as spallation neutron sources, heavy ion fusion, and nuclear waste transmutation, to mention a few examples. Charged particle beams are subject to various collective processes that can affect the beam quality. Of particular importance at the high beam currents and charge densities of practical interest are the effects of the intense self-fields produced by the beam space charge and current on determining detailed equilibrium, stability, and transport properties. In general, a complete description of collective processes in intense charged particle beams is provided by the three-dimensional Vlasov-Maxwell equations [1] for the self-consistent nonlinear evolution of the beam distribution function, $f_{b}(\mathbf{x}, \mathbf{p}, t)$, and the self-generated electric and magnetic fields, $\mathbf{E}^{s}(\mathbf{x}, t)$ and $\mathbf{B}^{s}(\mathbf{x}, t)$. While considerable progress has been made in analytical and numerical simulation studies of intense beam propagation [9-39], the effects of finite geometry and intense self-fields often make it difficult to obtain detailed predictions of beam equilibrium, stability, and transport properties based on the Vlasov-Maxwell equations. Nonetheless, often with the aid of numerical simulations, there has been considerable recent analytical progress in applying the VlasovMaxwell equations to investigate the detailed equilibrium and stability properties of intense charged particle beams. These investigations include a wide variety of
PACS numbers: 41.75.-i, 52.59.Sa, 52.35.-g, 52.59.Tb

applications ranging from the Harris-type instability driven by large temperature anisotropy with $T_{\perp b} \gg T_{\| b}$ [37], to the dipole-mode two-stream instability for an intense ion beam propagating through background electrons [29], to the resistive hose instability [34] and the sausage and hollowing instabilities [35] for intense beam propagation through background plasma, to the development of a nonlinear stability theorem $[22,23]$ in the smooth-focusing approximation.

While the collective processes described in the previous paragraph are three dimensional in nature, considerable theoretical progress has also been made in the development and application of one-dimensional Vlasov-Maxwell models [40-47] to describe the longitudinal beam dynamics for a long coasting beam, with applications ranging from plasma echo excitations to the investigation of coherent soliton structures, both compressional and rarefactive (holelike). Such one-dimensional Vlasov descriptions rely heavily on using a quasi-selfconsistent geometric-factor ( $g$-factor) model [46-55] to incorporate the average effects of the transverse beam geometry and the surrounding wall structure. Several limitations are evident in existing $g$-factor models. First, the models [40-55] typically assume a flattop (step-function) density profile in the plane perpendicular to beam propagation. While this is a good approximation for very-low-emittance, space-charge-dominated beams $[1,49-53]$, the case typically encountered in beams with low-to-moderate intensity is one in which the transverse density profile is bell shaped. Second, with the exception 
of the $g$-factor model for a step-function density profile developed by Reiser et al. [50-53], other $g$-factor models neglect the dependence of the beam edge radius $\left(r_{b}\right)$ and root-mean-square radius $\left(R_{b}\right)$ on the line density $\lambda_{b}$ of beam particles, an effect that becomes increasingly important at moderate and high beam intensities. Finally, some one-dimensional Vlasov treatments Taylor expand the field perturbations about the beam axis at $r=0$, an approximation which is not necessary and has questionable validity, particularly at moderate and high beam intensities.

The purpose of the present paper is to develop an improved one-dimensional kinetic model describing the self-consistent nonlinear evolution of the longitudinal distribution function $F_{b}\left(z, p_{z}, t\right)$ and average axial electric field $\left\langle E_{z}^{s}\right\rangle(z, t)$ for a very long charge bunch (coasting beam) propagating through a cylindrical conducting pipe with radius $r_{w}$, and confined in the transverse direction by an applied focusing force $\mathbf{F}_{\text {foc }}^{\text {tr }}$ described in the smoothfocusing approximation. For simplicity, to illustrate the basic approach, in the present analysis the cylindrical pipe is assumed to be perfectly conducting, and the beam transport geometry is assumed to be straight (linear). The analysis can be extended in a straightforward manner to include the effects of a conducting wall with finite resistive and capacitive impedance, as well as the effects of a slip factor $\eta$ in large-aspect-ratio circular geometry. Extension of the present analysis to incorporate these effects will be the subject of a future publication. In the present analysis, the coasting beam propagates in the $z$ direction with directed axial kinetic energy $\left(\gamma_{b}-1\right) m_{b} c^{2}$, where $\gamma_{b}=\left(1-\beta_{b}^{2}\right)^{-1 / 2}$ is the relativistic mass factor, $V_{b}=\beta_{b} c$ is the average axial velocity of the beam particles, $m_{b}$ is the rest mass of a beam particle, and $c$ is the speed of light in vacuo. Furthermore, the beam dynamics is treated in the thinbeam (paraxial) approximation, and the particle motions in the beam frame are assumed to be nonrelativistic.

For simplicity, the present analysis is carried out in the beam frame where the particle motions are nonrelativistic and the self-generated fields are assumed to have electrostatic polarization $\left(\nabla \times \mathbf{E}^{s}=0\right.$ and $\left.\mathbf{B}^{s}=0\right)$. The final results for the longitudinal Vlasov-Maxwell equations are then Lorentz transformed back to the laboratory frame, moving with axial velocity $-V_{b}=-\beta_{b} c$ relative to the average motion of the particles in the beam frame.

In Sec. II, the starting point is the fully nonlinear, three-dimensional Vlasov-Maxwell equations for the distribution function $f_{b}(\mathbf{x}, \mathbf{p}, t)$ and self-generated fields in the beam frame (unprimed variables). A reduced Vlasov equation for the longitudinal distribution function $F_{b}\left(z, p_{z}, t\right)=\int d x d y \int d p_{x} d p_{y} f_{b}(\mathbf{x}, \mathbf{p}, t)$ is obtained by projecting out (integrating over) the transverse phasespace variables $\left(\mathbf{x}_{\perp}, \mathbf{p}_{\perp}\right)$. Making the single ansatz so that the dependence of the distribution function $f_{b}(\mathbf{x}, \mathbf{p}, t)$ on axial momentum $p_{z}$ is factorable [Eq. (15)] leads to a closed system of equations describing the selfconsistent evolution of the longitudinal distribution function $F_{b}\left(z, p_{z}, t\right)$ and the average axial electric field $\left\langle E_{z}^{s}\right\rangle(z, t)$. Here, assuming axisymmetry in the transverse plane $(\partial / \partial \theta=0)$, the average $\langle\cdots\rangle$ denotes the weighted transverse spatial average over $n_{b}(r, z, t) / \lambda_{b}(z, t)$ defined in Eq. (26), where $n_{b}=\int d^{3} p f_{b}$ is the number density of beam particles, and $\lambda_{b}=\int d p_{z} F_{b}=\int d x d y n_{b}$ is the axial line density of beam particles.

In Sec. III, we assume that the beam dynamics is relatively quiescent in the transverse plane (no transverse instability or beam mismatch), and take the transverse density profile to have the fixed-shape form $n_{b}=$ $\left(\lambda_{b} / \pi r_{b}^{2}\right) f\left(r / r_{b}\right)$ where the shape function $f\left(r / r_{b}\right)=$ $(n+1)\left(1-r^{2} / r_{b}^{2}\right)^{n}$ for $0 \leq r<r_{b}$, and $f\left(r / r_{b}\right)=0$ for $r_{b}<r \leq r_{w}$. Here, $n=0,1,2, \ldots$ is an integer, with $n=$ 0 corresponding to a step-function density profile, and $r_{b}$ is the edge radius of the beam. Moreover, the root-meansquare beam radius $R_{b}=\left\langle r^{2}\right\rangle^{1 / 2}$ and edge radius $r_{b}$ generally depend on the line density through the radial force-balance condition in Eqs. (33) and (38), respectively. Of course, this dependence is weak $\left(r_{b} \simeq\right.$ const) for a low-intensity, emittance-dominated beam, whereas $r_{b}^{2} \propto \lambda_{b}$ for a very-low-emittance, space-chargedominated beam. Denoting $\partial / \partial z \sim L_{z}^{-1} \sim k_{z}$, we assume slow axial variations of $F_{b}\left(z, p_{z}, t\right)$ and $\lambda_{b}(z, t)$ with $k_{z}^{2} r_{w}^{2} \ll 1$. The average electric field $\left\langle E_{z}^{s}\right\rangle(z, t)$, expressed in terms of $\partial \lambda_{b} / \partial z$ and higher-order derivatives, together with closed forms for the corresponding geometric factors are calculated self-consistently correct to $0\left(k_{z}^{2} r_{w}^{2}\right)$ for the class of bell-shaped density profiles with $n=$ $0,1,2, \ldots$ described above. The results show a strong dependence of the geometric factors on profile shape and beam intensity $\lambda_{b}$.

In Sec.IV, as a simple application, the resulting coupled equations for the longitudinal distribution function $F_{b}\left(z, p_{z}, t\right)$ and the average electric field $\left\langle E_{z}^{s}\right\rangle(z, t)$ are solved in the linearization approximation for the case of low-to-moderate beam intensity treating $r_{b} \simeq$ const (independent of $\lambda_{b}$ ). As expected, the analysis leads to collective oscillations with sound-wave-like characteristics modified by cubic dispersion. The detailed oscillation and damping properties of the perturbation of course depends on the choice of equilibrium distribution function $F_{b}^{0}\left(p_{z}\right)$ about which the system is perturbed.

Finally, in Sec. V, the key results derived in the beam frame are transformed back to the laboratory frame to facilitate practical applications of the improved kinetic model of the longitudinal beam dynamics developed here.

\section{THEORETICAL MODEL AND ONE- DIMENSIONAL VLASOV EQUATION}

The present analysis considers a very long charge bunch (coasting beam) with characteristic axial length 
$l_{b}$ and radius $r_{b}$ satisfying $l_{b} \gg r_{b}$. The coasting beam is made up of particles with charge $e_{b}$ and rest mass $m_{b}$ propagating in the $z$ direction with directed axial kinetic energy $\left(\gamma_{b}-1\right) m_{b} c^{2}$, where $\gamma_{b}=\left(1-\beta_{b}^{2}\right)^{-1 / 2}$ is the relativistic mass factor, $V_{b}=\beta_{b} c$ is the average axial velocity of the beam particles, and $c$ is the speed of light in vacuo. It is assumed that the beam propagates through a straight, perfectly conducting cylindrical pipe with wall radius $r_{w}$, and the applied transverse focusing force $\mathbf{F}_{\text {foc }}^{\mathrm{tr}}$ is modeled in the smooth-focusing approximation. For example, one simple model is $\mathbf{F}_{\text {foc }}^{\mathrm{tr}}=-\gamma_{b} m_{b} \omega_{\beta \perp}^{2} \mathbf{x}_{\perp}$, where $\omega_{\beta \perp}=$ const is the average focusing frequency associated with the applied focusing field, and $\mathbf{x}_{\perp}$ is the transverse displacement from the cylinder axis. Finally, the nonlinear dynamics of the beam particles is treated in the thin-beam (paraxial) approximation, and the particle motions in the beam frame are assumed to be nonrelativistic [1].

The most general description of the nonlinear collective interactions in an intense charged particle beam is provided by the nonlinear Vlasov-Maxwell equations [1], which is the theoretical framework adopted in the present analysis. For simplicity, the analysis is carried out in the beam frame $\left(V_{b}=\beta_{b} c=0\right.$ and $\left.\gamma_{b}=1\right)$, and the kinetic description is based on the Vlasov-Maxwell equations, which describe the self-consistent nonlinear evolution of the distribution function $f_{b}(\mathbf{x}, \mathbf{p}, t)$ and the self-generated electric and magnetic fields, $\mathbf{E}^{s}(\mathbf{x}, t)$ and $\mathbf{B}^{s}(\mathbf{x}, t)$, in the six-dimensional phase space $(\mathbf{x}, \mathbf{p})$. Here, the (unprimed) variables $(\mathbf{x}, \mathbf{p}, t)$ denote beam-frame variables. For present purposes, the self-generated fields in the beam frame are assumed to have longitudinal polarization (electrostatic approximation) with $\nabla \times \mathbf{E}^{s}=0$ and $\mathbf{B}^{s}=0$. In this case, the nonlinear Vlasov-Maxwell equations describing the self-consistent nonlinear evolution of $f_{b}(\mathbf{x}, \mathbf{p}, t)$ and $\mathbf{E}^{s}(\mathbf{x}, t)$ can be expressed as [1]

$$
\frac{\partial f_{b}}{\partial t}+\mathbf{v} \cdot \frac{\partial f_{b}}{\partial \mathbf{x}}+\left(\mathbf{F}_{\mathrm{foc}}^{\mathrm{tr}}+e_{b} \mathbf{E}^{s}\right) \cdot \frac{\partial f_{b}}{\partial \mathbf{p}}=0,
$$

and

$$
\nabla \cdot \mathbf{E}^{s}=4 \pi e_{b} \int d^{3} p f_{b}, \quad \nabla \times \mathbf{E}^{s}=0,
$$

where $\mathbf{v}=\mathbf{p} / m_{b}$ is the (nonrelativistic) particle velocity in the beam frame. Of course, once the solutions for $f_{b}(\mathbf{x}, \mathbf{p}, t)$ and $\mathbf{E}^{s}(\mathbf{x}, t)$ are determined from Eqs. (1) and (2) in the beam frame, the corresponding solutions in the laboratory frame (moving with axial velocity $-V_{b} \hat{\mathbf{e}}_{z}$ relative to the beam frame) can be obtained by transforming the phase-space variables and field components to the laboratory frame (primed variables) according to [22]

$$
\begin{aligned}
& x^{\prime}=x, \quad y^{\prime}=y, \quad z^{\prime}=\gamma_{b}\left(z+V_{b} t\right), \quad p_{x}^{\prime}=p_{x}, \\
& p_{y}^{\prime}=p_{y}, \quad p_{z}^{\prime}=\gamma_{b}\left(p_{z}+\gamma m_{b} V_{b}\right), \\
& t^{\prime}=\gamma_{b}\left(t+V_{b} z / c^{2}\right), \quad \gamma^{\prime}=\gamma_{b}\left(\gamma+V_{b} p_{z} / m_{b} c^{2}\right),
\end{aligned}
$$

and

$$
\begin{aligned}
& {\left[E_{x}^{s}\right]^{\prime}=\gamma_{b} E_{x}^{s}, \quad\left[E_{y}^{s}\right]^{\prime}=\gamma_{b} E_{y}^{s}, \quad\left[E_{z}^{s}\right]^{\prime}=E_{z}^{s},} \\
& {\left[B_{x}^{s}\right]^{\prime}=-\frac{1}{c} \gamma_{b} V_{b} E_{y}^{s}, \quad\left[B_{y}^{s}\right]^{\prime}=\frac{1}{c} \gamma_{b} V_{b} E_{x}^{s},} \\
& {\left[B_{z}^{s}\right]^{\prime}=0 .}
\end{aligned}
$$

In Eq. (3), $\gamma^{\prime}=\left(1+\mathbf{p}^{2} / m_{b}^{2} c^{2}\right)^{1 / 2} \quad$ and $\quad \gamma=(1+$ $\left.\mathbf{p}^{2} / m_{b}^{2} c^{2}\right)^{1 / 2}$ are the kinematic mass factors, and $\gamma \simeq 1+$ $\mathbf{p}^{2} / 2 m_{b}^{2} c^{2}$ because the particle motions in the beam frame are assumed to be nonrelativistic.

Returning to the beam frame, the Vlasov-Poisson equations (1) and (2) are fully three dimensional and describe the self-consistent nonlinear evolution of the distribution function $f_{b}(\mathbf{x}, \mathbf{p}, t)$ in the transverse phase space $\left(\mathbf{x}_{\perp}, \mathbf{p}_{\perp}\right)=\left(x, y, p_{x}, p_{y}\right)$ and longitudinal phase space $\left(z, p_{z}\right)$. Two macroscopic moments of particular interest for a coasting beam (long charge bunch) are the volume number density $n_{b}(x, y, z, t)$ and axial line density $\lambda_{b}(z, t)$ defined by

$$
\begin{aligned}
& n_{b}(\mathbf{x}, t)=\int d^{3} p f_{b}(\mathbf{x}, \mathbf{p}, t), \\
& \lambda_{b}(z, t)=\int d x d y n_{b}(\mathbf{x}, t)=\int d x d y \int d^{3} p f_{b} .
\end{aligned}
$$

Here, $\int d x d y \cdots=\int_{0}^{r_{w}} d r r \int_{0}^{2 \pi} d \theta \cdots$ denotes integration over the accessible transverse configuration space extending to the conducting wall located at radius $r=$ $r_{w}, \int d^{3} p \cdots=\int_{-\infty}^{\infty} d p_{x} \int_{-\infty}^{\infty} d p_{y} \int_{-\infty}^{\infty} d p_{z} \cdots$ denotes integration over momentum space, and $(r, \theta, z)$ are cylindrical polar coordinates with $x=r \cos \theta$ and $y=r \sin \theta$, where $r=\left(x^{2}+y^{2}\right)^{1 / 2}$ is the radial distance from the beam axis at $(x, y)=(0,0)$. Because the cylindrical wall located at $r=r_{w}$ is perfectly conducting, the nonlinear Vlasov-Poisson equations are to be solved subject to the boundary conditions

$$
\left[E_{z}\right]_{r=r_{w}}=0=\left[E_{\theta}\right]_{r=r_{w}} .
$$

Moreover, the present analysis, carried out for a long coasting beam, assumes that the distribution function $f_{b}(\mathbf{x}, \mathbf{p}, t)$ is such that the transverse beam dynamics is relatively quiescent (e.g., no transverse instability), and that there are no particles extending beyond some outer radius $r_{0}<r_{w}$, i.e.,

$$
f_{b}(\mathbf{x}, \mathbf{p}, t)=0, \quad \text { for } r_{0}<r \leq r_{w} .
$$

That is, there is a well-defined vacuum region outside the beam with zero number density, $n_{b}=0$ in the region $r_{0}<$ $r \leq r_{w}$ [see Eqs. (5) and (7)]. Finally, it is assumed that

$$
\begin{aligned}
& f_{b}(\mathbf{x}, \mathbf{p}, t)=0, \quad \text { for } p_{x} \rightarrow \pm \infty, \\
& p_{y} \rightarrow \pm \infty, \quad \text { or } \quad p_{z} \rightarrow \pm \infty,
\end{aligned}
$$

which is consistent with the existence of the density integral defined in Eq. (5).

For purposes of deriving a one-dimensional Vlasov equation describing the beam dynamics in the longitudinal phase space $\left(z, p_{z}\right)$, it is convenient to rewrite the three-dimensional Vlasov equation (1) describing the 
nonlinear evolution of $f_{b}(\mathbf{x}, \mathbf{p}, t)$ in the equivalent form

$$
\begin{aligned}
\frac{\partial}{\partial t} f_{b} & +v_{z} \frac{\partial}{\partial z} f_{b}+\mathbf{v}_{\perp} \cdot \frac{\partial}{\partial \mathbf{x}_{\perp}} f_{b}+\left(\mathbf{F}_{\mathrm{foc}}^{\mathrm{tr}}+e_{b} \mathbf{E}_{\perp}^{s}\right) \cdot \frac{\partial}{\partial \mathbf{p}_{\perp}} f_{b} \\
& +e_{b} E_{z}^{s} \frac{\partial}{\partial p_{z}} f_{b}=0 .
\end{aligned}
$$

Here, $\mathbf{x}_{\perp}=x \hat{\mathbf{e}}_{x}+y \hat{\mathbf{e}}_{y}$ and $\mathbf{p}_{\perp}=p_{x} \hat{\mathbf{e}}_{x}+p_{y} \hat{\mathbf{e}}_{y}$ denote the transverse phase-space variables, and $\mathbf{v}_{\perp}=\mathbf{p}_{\perp} / m_{b}$ is the transverse particle velocity. We introduce the transverse projection operator $\langle\cdots\rangle_{\perp}$ defined by

$$
\langle\cdots\rangle_{\perp}=\int d x d y \int d p_{x} d p_{y} \cdots,
$$

where $\quad \int d x d y \cdots=\int_{0}^{2 \pi} d \theta \int_{0}^{r_{w}} d r r \cdots$ and $\int d p_{x} d p_{y} \cdots=\int_{-\infty}^{\infty} d p_{x} \int_{-\infty}^{\infty} d p_{y} \cdots$. Note that the operator defined in Eq. (10) has the effect of projecting out the transverse phase-space coordinates $\left(\mathbf{x}_{\perp}, \mathbf{p}_{\perp}\right)$. Introducing the longitudinal distribution function $F_{b}\left(z, p_{z}, t\right)$ defined by

$$
\begin{aligned}
F_{b}\left(z, p_{z}, t\right) & =\left\langle f_{b}(\mathbf{x}, \mathbf{p}, t)\right\rangle_{\perp} \\
& =\int d x d y \int d p_{x} d p_{y} f_{b}(\mathbf{x}, \mathbf{p}, t),
\end{aligned}
$$

we operate on Eq. (9) with $\int d x d y \int d p_{x} d p_{y} \cdots$. Some straightforward integration by parts that makes use of the boundary conditions in Eqs. (7) and (8) readily gives for the nonlinear evolution of $F_{b}\left(z, p_{z}, t\right)$

$$
\frac{\partial}{\partial t} F_{b}+v_{z} \frac{\partial}{\partial z} F_{b}+e_{b}\left\langle E_{z}^{s} \frac{\partial}{\partial p_{z}} f_{b}\right\rangle_{\perp}=0 .
$$

Note that $E_{z}^{s}(\mathbf{x}, t)$ occurs in the average in Eq. (12). We operate on Poisson's equation $\nabla \cdot \mathbf{E}^{s}=4 \pi e_{b} \int d^{3} p f_{b}=$ $4 \pi e_{b} n_{b}$ in Eq. (2) with $\partial / \partial z$, and make use of $\nabla \times \mathbf{E}^{s}=0$ in cylindrical polar coordinates, which gives $\partial E_{z}^{s} / \partial r=\partial E_{r}^{s} / \partial z$ and $\partial E_{\theta}^{s} / \partial z=r^{-1} \partial E_{z}^{s} / \partial \theta$. Poisson's equation for $E_{z}^{s}(r, \theta, z, t)$ readily becomes

$$
\frac{1}{r} \frac{\partial}{\partial r} r \frac{\partial}{\partial r} E_{z}^{s}+\frac{\partial^{2}}{\partial z^{2}} E_{z}^{s}+\frac{1}{r^{2}} \frac{\partial^{2}}{\partial \theta^{2}} E_{z}^{s}=4 \pi e_{b} \frac{\partial}{\partial z} n_{b},
$$

where $n_{b}(r, \theta, z, t)=\int d^{3} p f_{b}(r, \theta, z, \mathbf{p}, t)$ is the number density of beam particles. In Eqs. (12) and (13) we note from Eqs. (5) and (11) that the axial line density $\lambda_{b}(z, t)=\int d x d y n_{b}=\int d x d y \int d^{3} p f_{b}$ is related (exactly) to the longitudinal distribution function $F_{b}\left(z, p_{z}, t\right)$ by

$$
\lambda_{b}(z, t)=\int d p_{z} F_{b}\left(z, p_{z}, t\right)
$$

The Vlasov equation (12) for the evolution of $F_{b}\left(z, p_{z}, t\right)$ contains the average $e_{b}\left\langle E_{z}^{s} \partial f_{b} \partial p_{z}\right\rangle_{\perp}$ over the three-dimensional distribution function $f_{b}(\mathbf{x}, \mathbf{p}, t)$. Of course the total distribution function $f_{b}(\mathbf{x}, \mathbf{p}, t)$ evolves according to the nonlinear Vlasov equation (1), which generally couples the longitudinal and transverse particle dynamics. As a simplifying ansatz for closure, consistent with the assumption that the transverse beam dynamics remains relatively quiescent, we make the assumption that the dependence of $f_{b}(\mathbf{x}, \mathbf{p}, t)$ on axial momentum $p_{z}$ is factorable according to

$$
f_{b}(\mathbf{x}, \mathbf{p}, t)=G_{b}\left(\mathbf{x}_{\perp}, \mathbf{p}_{\perp}, z, t\right) F_{b}\left(z, p_{z}, t\right),
$$

where $G_{b}\left(\mathbf{x}_{\perp}, \mathbf{p}_{\perp}, z, t\right)$ and $F_{b}\left(z, p_{z}, t\right)$ are the transverse and longitudinal distribution functions, respectively. From Eqs. (5), (11), and (15), we readily obtain

$$
\int d p_{x} d p_{y} G_{b}\left(\mathbf{x}_{\perp}, \mathbf{p}_{\perp}, z, t\right)=\frac{n_{b}(\mathbf{x}, t)}{\lambda_{b}(z, t)} .
$$

Substituting Eqs. (15) and (16) into the definition of $e_{b}\left\langle E_{z}^{s} \partial f_{b} / \partial p_{z}\right\rangle_{\perp}$ then gives

$$
e_{b}\left\langle E_{z}^{s} \frac{\partial}{\partial p_{z}} f_{b}\right\rangle_{\perp}=e_{b}\left\langle E_{z}^{s}\right\rangle(z, t) \frac{\partial}{\partial p_{z}} F_{b}\left(z, p_{z}, t\right),
$$

where the average axial electric field $\left\langle E_{z}^{s}\right\rangle(z, t)$ is defined by

$$
\begin{aligned}
\left\langle E_{z}^{s}\right\rangle(z, t) & \equiv\left\langle E_{z}^{s}(\mathbf{x}, t) G_{b}\left(\mathbf{x}_{\perp}, \mathbf{p}_{\perp}, z, t\right)\right\rangle_{\perp} \\
& =\int d x d y E_{z}^{s}(\mathbf{x}, t) \frac{n_{b}(\mathbf{x}, t)}{\lambda_{b}(z, t)} .
\end{aligned}
$$

Here, use has been made of the definition of the perpendicular projection operator $\langle\cdots\rangle_{\perp}$ in Eq. (10). From Eqs. (17) and (18), an important consequence of the factorability ansatz in Eq. (15) is that the average electric field $\left\langle E_{z}\right\rangle(z, t)$ occurring in the longitudinal Vlasov equation (12) corresponds to a transverse spatial average of $E_{z}^{s}(\mathbf{x}, t)$ appropriately weighted by the density profile of the beam particles.

In the remainder of this paper we specialize to the case where all beam and field quantities are assumed to be axisymmetric $(\partial / \partial \theta=0)$. Making use of Eqs. (12), (13), and (18), the final set of equations describing the nonlinear evolution of $F_{b}\left(z, p_{z}, t\right)$ and $\left\langle E_{z}^{s}\right\rangle(z, t)$ are given by

$$
\frac{\partial}{\partial t} F_{b}+v_{z} \frac{\partial}{\partial z} F_{b}+e_{b}\left\langle E_{z}^{s}\right\rangle \frac{\partial}{\partial p_{z}} F_{b}=0,
$$

and

$$
\left\langle E_{z}^{s}\right\rangle=2 \pi \int_{0}^{r_{w}} d r r \frac{n_{b}(r, z, t)}{\lambda_{b}(z, t)} E_{z}^{s}(r, z, t) .
$$

Here, $E_{z}^{s}(r, z, t)$ is determined self-consistently in terms of the density profile $n_{b}(r, z, t)$ from Poisson's equation (13), which can be expressed as

$$
\frac{1}{r} \frac{\partial}{\partial r} r \frac{\partial}{\partial r} E_{z}^{s}+\frac{\partial^{2}}{\partial z^{2}} E_{z}^{s}=4 \pi e_{b} \frac{\partial}{\partial z} n_{b}
$$

for $\partial / \partial \theta=0$. In addition, from Eqs. (5) and (14), the line density $\lambda_{b}(z, t)$ is related to $F_{b}\left(z, p_{z}, t\right)$ and $n_{b}(r, z, t)$ by 


$$
\lambda_{b}(z, t)=2 \pi \int_{0}^{r_{w}} d r r n_{b}(r, z, t)=\int d p_{z} F_{b}\left(z, p_{z}, t\right) .
$$

Equations (19)-(22) constitute the final form of the nonlinear Vlasov-Maxwell equations describing the evolution of the longitudinal distribution function $F_{b}\left(z, p_{z}, t\right)$ and line density $\lambda_{b}(z, t)$ in a long coasting beam. An important feature is that Eqs. (19)-(22) have been derived for general radial dependence of the density profile $n_{b}(r, z, t)$. The procedure for the solution is the following. Once the radial dependence of $n_{b}(r, z, t)$ is specified, $E_{z}^{s}(r, z, t)$ is calculated from Poisson's equation (21). The resulting expression for $E_{z}^{s}(r, z, t)$ is then substituted into Eq. (20) to determine the average electric field $\left\langle E_{z}^{s}\right\rangle(z, t)$, which in turn is used in the Vlasov equation (19) to determine the self-consistent nonlinear evolution of $F_{b}\left(z, p_{z}, t\right)$. Most importantly, the average electric field $\left\langle E_{z}^{s}\right\rangle(z, t)$ occurring in Eqs. (19) and (20) is appropriately weighted by a transverse spatial average over the density profile of the beam particles. No a priori assumption has been made that the density profile corresponds to a flattop (step-function) distribution in the radial direction [4053]. Nor have we Taylor expanded Poisson's equation (21) about $r=0$ to estimate the average electric field $\left\langle E_{z}^{s}\right\rangle(z, t)$ [46,47]. Indeed, Eqs. (19)-(22) are valid for the general choice of the radial density profile and can be applied for arbitrary beam intensity ranging from low-intensity, emittance-dominated beams to very-high-intensity, space-charge-dominated beams.

\section{EVALUATION OF AVERAGE LONGITUDINAL FIELD $\left\langle\boldsymbol{E}_{\boldsymbol{Z}}^{S}\right\rangle$}

Equations (19)-(22) constitute the final form of the Vlasov-Maxwell equations describing the nonlinear evolution of the distribution function $F_{b}\left(z, p_{z}, t\right)$ and line density $\lambda_{b}(z, t)$. In this section, we make use of Eqs. (20) and (21) to derive a simplified expression for the average longitudinal electric field $\left\langle E_{z}^{s}\right\rangle(z, t)$, valid for a wide range of choices of bell-shaped density profiles. For a specified functional form of $n_{b}(r, z, t)$, Eq. (21) can, of course, be formally solved for $E_{z}^{s}(r, z, t)$ using a Green's function method [50,51]. For our purposes here, however, we specialize to the case where the $z$ variation in $E_{z}^{s}(r, z, t)$ is slow in comparison with the $r$ variation. In particular, denoting $\partial / \partial z \sim L_{z}^{-1} \sim k_{z}$, it is assumed that

$$
k_{z}^{2} r_{w}^{2} \ll 1,
$$

where $r_{w}$ is the conducting wall radius.

\section{A. Average electric field $\left\langle E_{z}^{s}\right\rangle$ for $k_{z}^{2} r_{w}^{2} \ll 1$}

Imposing the boundary condition $E_{z}^{s}\left(r=r_{w}, z, t\right)=0$ at the perfecting conducting wall [Eq. (6)], the formal solution to Eq. (21) can be expressed as

$$
E_{z}^{s}(r, z, t)=-4 \pi e_{b} \int_{r}^{r_{w}} \frac{d r}{r} \int_{0}^{r} d r r\left(\frac{\partial n_{b}}{\partial z}-\frac{1}{4 \pi e_{b}} \frac{\partial^{2} E_{z}^{s}}{\partial z^{2}}\right),
$$

where $n_{b}(r, z, t)$ is the density profile. The second term on the right-hand side of Eq. (24) is small in comparison with the first term by virtue of the inequality in Eq. (23). Solving iteratively for $E_{z}^{s}(r, z, t)$ then gives

$E_{z}^{s}(r, z, t)=-4 \pi e_{b} \int_{r}^{r_{w}} \frac{d r}{r} \int_{0}^{r} d r r\left(\frac{\partial n_{b}}{\partial z}+\int_{r}^{r_{w}} \frac{d r}{r} \int_{0}^{r} d r r \frac{\partial^{3} n_{b}}{\partial z^{3}}\right)$

correct to $0\left(k_{z}^{2} r_{w}^{2}\right)$. Equation (25) is the degree of accuracy used for the longitudinal electric field $E_{z}^{s}(r, z, t)$ in the subsequent analysis. Substituting Eq. (25) into Eq. (20) and performing a straightforward integration by parts gives

$$
\begin{aligned}
\left\langle E_{z}^{s}\right\rangle & =-2 \pi e_{b} \int_{0}^{r_{w}} d r r \frac{n_{b}}{\lambda_{b}} \int_{r}^{r_{w}} \frac{d r}{r}\left[\frac{\partial}{\partial z}\left(2 \pi \int_{0}^{r} d r r n_{b}\right)+\frac{\partial^{3}}{\partial z^{3}} \int_{0}^{r} d r r \int_{r}^{r_{w}} \frac{d r}{r}\left(2 \pi \int_{0}^{r} d r r n_{b}\right)\right] \\
& =-2 e_{b} \int_{0}^{r_{w}} \frac{d r}{r}\left(2 \pi \int_{0}^{r} d r r \frac{n_{b}}{\lambda_{b}}\right)\left[\frac{\partial}{\partial z}\left(2 \pi \int_{0}^{r} d r r n_{b}\right)+\frac{\partial^{3}}{\partial z^{3}} \int_{0}^{r} d r r \int_{r}^{r_{w}} \frac{d r}{r}\left(2 \pi \int_{0}^{r} d r r n_{b}\right)\right] .
\end{aligned}
$$

Equation (26) can be expressed in the equivalent form

where $\left\langle E_{z}^{s}\right\rangle^{(1)}$ and $\left\langle E_{z}^{s}\right\rangle^{(2)}$ are defined by

$$
\left\langle E_{z}^{s}\right\rangle=\left\langle E_{z}^{s}\right\rangle^{(1)}+\left\langle E_{z}^{s}\right\rangle^{(2)},
$$

$$
\left\langle E_{z}^{s}\right\rangle^{(1)}=-2 e_{b} \int_{0}^{r_{w}} \frac{d r}{r}\left(2 \pi \int_{0}^{r} d r r \frac{n_{b}}{\lambda_{b}}\right) \frac{\partial}{\partial z}\left(2 \pi \int_{0}^{r} d r r \frac{n_{b}}{\lambda_{b}} \cdot \lambda_{b}\right),
$$

and

$$
\left\langle E_{z}^{s}\right\rangle^{(2)}=-2 e_{b} \int_{0}^{r_{w}} \frac{d r}{r}\left(2 \pi \int_{0}^{r} d r r \frac{n_{b}}{\lambda_{b}}\right) \frac{\partial^{3}}{\partial z^{3}} \int_{0}^{r} d r r \int_{r}^{r_{w}} \frac{d r}{r}\left(2 \pi \int_{0}^{r} d r r \frac{n_{b}}{\lambda_{b}} \cdot \lambda_{b}\right) .
$$

Equations (27)-(29) can be used to evaluate a closed expression for $\left\langle E_{z}^{s}\right\rangle=\left\langle E_{z}^{s}\right\rangle^{(1)}+\left\langle E_{z}^{s}\right\rangle^{(2)}$ and the corresponding $g$ 
factors for a wide range of choices of beam density profiles $n_{b}(r, z, t)$. Note from Eqs. (28) and (29) that $\left\langle E_{z}^{s}\right\rangle^{(2)}$ is of order $k_{z}^{2} r_{w}^{2} \ll 1$ smaller than $\left\langle E_{z}^{s}\right\rangle^{(1)}$ by virtue of the assumption in Eq. (23).

\section{B. Fixed-shape density profiles $n_{b}(r, z, t)$}

A wide variety of functional forms for the density profile $n_{b}(r, z, t)$ can be used to evaluate $\left\langle E_{z}^{s}\right\rangle$ from Eqs. (27)-(29). For example, one approach is to take the transverse distribution function to correspond to the class of matched-beam quasiequilibrium distributions of the form $G_{b}\left(H_{\perp}\right)$ [1], where $H_{\perp}=p_{\perp}^{2} / 2 m_{b}+m_{b} \omega_{\beta \perp}^{2} r^{2} / 2+$ $e_{b} \phi$ is the Hamiltonian for transverse particle motion, and $\phi$ is the space-charge potential determined from Poisson's equation. In this case, the density profile is determined from $n_{b} / \lambda_{b}=2 \pi \int_{0}^{\infty} d p_{\perp} p_{\perp} G_{b}\left(H_{\perp}\right)$ [Eq. (16)]. For specified functional form of $G_{b}\left(H_{\perp}\right)$, the radial dependence of the density profile can be determined self-consistently from Poisson's equation $r^{-1}(\partial / \partial r)[r \partial \phi / \partial r]=-4 \pi e_{b} n_{b}$ for weak variations with axial coordinate $z$. Depending on the choice of $G_{b}\left(H_{\perp}\right)$, the radial density profile ranges from a step-function profile for a Kapchinskij-Vladimirskij distribution [1,9-11], to a bell-shaped profile with diffuse radial boundary for a thermal equilibrium distribution $[1,2]$, to a bell-shaped profile with a sharp radial boundary for a waterbag distribution $[1,14,15,38]$. Whatever the choice of $G_{b}\left(H_{\perp}\right)$, the mean-square beam radius $R_{b}^{2}=\left\langle r^{2}\right\rangle \equiv\left[4 \pi^{2} \int_{0}^{r_{w}} d r r r^{2} \int_{0}^{\infty} d p_{\perp} p_{\perp} G_{b}\left(H_{\perp}\right)\right] /$ $\left[4 \pi^{2} \int_{0}^{r_{w}} d r r \int_{0}^{\infty} d p_{\perp} p_{\perp} G_{b}\left(H_{\perp}\right)\right]$ is determined exactly for a matched beam from the radial force balance equation [1]

$$
\omega_{\beta \perp}^{2} R_{b}^{2}=\lambda_{b} \frac{e_{b}^{2}}{m_{b}}+\frac{1}{4} \frac{\tilde{\epsilon}^{2}}{R_{b}^{2}} .
$$

Here, the scaled emittance $\tilde{\boldsymbol{\epsilon}}$ in the beam frame is defined by $\tilde{\boldsymbol{\epsilon}}^{2} / 4 R_{b}^{2}=\left\langle v_{\perp}^{2}\right\rangle \equiv\left[4 \pi^{2} \int_{0}^{r_{w}} d r r \int_{0}^{\infty} d p_{\perp} p_{\perp}\left(p_{\perp}\right)\right.$ $\left.\left.m_{b}\right)^{2} G_{b}\left(H_{\perp}\right)\right] /\left[4 \pi^{2} \int_{0}^{r_{w}^{\perp}} d r r \int_{0}^{\infty} d p_{\perp} p_{\perp} G_{b}\left(H_{\perp}\right)\right]$.

The radial force balance equation (30) can be expressed as

$$
R_{b}^{4}-R_{\lambda}^{2} R_{b}^{2}-R_{\epsilon}^{4}=0
$$

where

$$
R_{\lambda}^{2}=\frac{\lambda_{b} e_{b}^{2}}{m_{b} \omega_{\beta \perp}^{2}}, \quad R_{\epsilon}^{4}=\frac{\tilde{\boldsymbol{\epsilon}}^{2}}{4 \omega_{\beta \perp}^{2}} .
$$

The physically acceptable solution to Eq. (31) is given by

$$
R_{b}^{2}=\frac{1}{2}\left[R_{\lambda}^{2}+\left(R_{\lambda}^{4}+4 R_{\epsilon}^{4}\right)^{1 / 2}\right]
$$

Note from Eqs. (32) and (33) that the mean-square beam radius $R_{b}^{2}$ depends on the line density $\lambda_{b}$. For a lowintensity emittance-dominated beam with $R_{\lambda}^{2} \ll 2 R_{\epsilon}^{2}$, Eq. (33) reduces to the familiar result $R_{b}^{2} \simeq R_{\epsilon}^{2}=$ $\tilde{\boldsymbol{\epsilon}} / 2 \omega_{\beta \perp}$, which is independent of $\lambda_{b}$. On the other hand, for a very-low-emittance, space-charge-dominated beam with $R_{\lambda}^{2} \gg 2 R_{\epsilon}^{2}$, Eq. (33) reduces to $R_{b}^{2} \simeq R_{\lambda}^{2}=$ $\lambda_{b} e_{b}^{2} / m_{b} \omega_{\beta \perp}^{2}$, which is linearly proportional to the line density $\lambda_{b}$. A plot of $R_{b}^{2} / R_{\epsilon}^{2}$ versus $R_{\lambda}^{2} / R_{\epsilon}^{2}$ illustrating this behavior is shown in Fig. 1.

To obtain a closed expression for $\left\langle E_{z}^{s}\right\rangle$ from Eqs. (27)(29), for our purposes here we specialize to the class of fixed-shape density profiles $n_{b}(r, z, t)$ of the form

$$
n_{b}= \begin{cases}\frac{\lambda_{b}}{\pi r_{b}^{2}} f\left(\frac{r}{r_{b}}\right), & 0 \leq r<r_{b} \\ 0, & r_{b}<r \leq r_{w}\end{cases}
$$

where $f\left(r / r_{b}\right)$ is a smooth function that depends on the scaled radial variable $r / r_{b}$, and $\lambda_{b}(z, t)=$ $2 \pi \int_{0}^{r_{w}} d r r n_{b}(r, z, t)$ is the line density of beam particles. Note from Eq. (34) that the density profile $n_{b}(r, z, t)$ has a sharp radial edge at $r=r_{b}$, and that there is a distinct vacuum region outside the beam $\left(r_{b}<r \leq r_{w}\right)$. From $\lambda_{b}=2 \pi \int_{0}^{r_{w}} d r r n_{b}$, we obtain from Eq. (34) the normalization condition

$$
\int_{0}^{1} d X X f(X)=\frac{1}{2}
$$

Similarly, from the definition of mean-square beam radius $R_{b}^{2}=\left\langle r^{2}\right\rangle=\left(2 \pi \int_{0}^{r_{w}} d r r r^{2} n_{b}\right) / \lambda_{b}$, we readily obtain

$$
R_{b}^{2}=\eta_{b} r_{b}^{2}
$$

where the constant $\eta_{b}$ is defined by

$$
\eta_{b}=2 \int_{0}^{1} d X X X^{2} f(X) .
$$

For specified shape function $f\left(r / r_{b}\right)$, we note from Eqs. (33) and (36) that the edge radius $r_{b}$ can be expressed as

$$
r_{b}^{2}=\frac{1}{2 \eta_{b}}\left[R_{\lambda}^{2}+\left(R_{\lambda}^{4}+4 R_{\epsilon}^{4}\right)^{1 / 2}\right]
$$

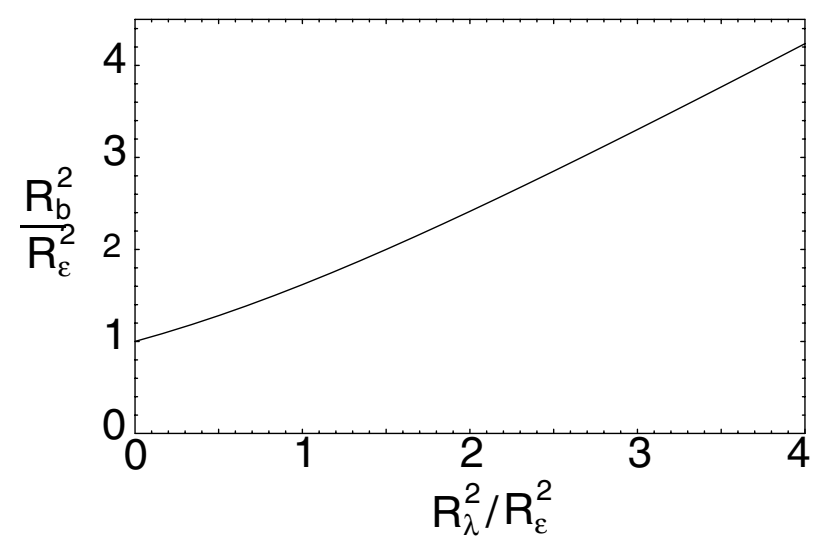

FIG. 1. Plot of $R_{b}^{2} / R_{\epsilon}^{2}$ versus $R_{\lambda}^{2} / R_{\epsilon}^{2}$ obtained from the radial force balance Eq. (33). 
where $R_{\lambda}^{2}$ and $R_{\epsilon}^{4}$ are defined in Eq. (32), and the constant $\eta_{b}$ is defined in Eq. (37).

There are many practical choices of density shape function $f\left(r / r_{b}\right)$ in Eq. (34). One form of particular interest is the profile

$$
f_{n}\left(\frac{r}{r_{b}}\right)=(n+1)\left[1-\frac{r^{2}}{r_{b}^{2}}\right]^{n}, \quad 0 \leq r<r_{b},
$$

where $n=0,1,2, \ldots$ is a positive integer, and the normalization of $f_{n}\left(r / r_{b}\right)$ in Eq. (39) satisfies Eq. (35). For the profile in Eq. (39), we readily obtain from Eq. (37) that

$$
\eta_{b}=\frac{1}{n+2}
$$

where $n=0,1,2, \ldots$ Here, the root-mean-square beam radius $R_{b}$ is related to the edge radius $r_{b}$ by $R_{b}^{2}=\eta_{b} r_{b}^{2}$. The profile shape function $f\left(r / r_{b}\right)$ defined in Eq. (39) gives a wide range of density profile peakedness, ranging from a step-function density profile (for $n=0$ ) to increasingly peaked profiles (for $n=1,2, \ldots$ ). The two cases corresponding to $n=0$ and $n=2$ are illustrated in Fig. 2. For $n=2$, note from Fig. 2 and Eqs. (34) and (39) that the bell-shaped density profile $n_{b}$ approaches zero continuously at $r=r_{b}$ with $\left[n_{b}\right]_{r=r_{b}}=0=$ $\left[\partial n_{b} / \partial r\right]_{r=r_{b}}$.

We reiterate that the validity of the one-dimensional kinetic model consisting of Eqs. (19), (22), and (26) requires that the beam dynamics remains relatively quiescent in the transverse plane (no transverse instability or beam mismatch). In this regard, it is important to recognize that a sufficient condition for transverse stability of matched-beam quasiequilibrium distributions of the form $G_{b}\left(H_{\perp}\right)$ is given by $\partial G_{b}\left(H_{\perp}\right) / \partial H_{\perp} \leq 0$ [see, for example, Chapters 4 and 7 of Ref. [1]]. Here $G_{b}\left(H_{\perp}\right)$ is the transverse distribution function, $H_{\perp}=p_{\perp}^{2} / 2 m_{b}+$ $m_{b} \omega_{\beta \perp}^{2} r^{2} / 2+e_{b} \phi(r)$ is the Hamiltonian for transverse particle motion, and $\phi$ is the space-charge potential. The condition $\partial G_{b}\left(H_{\perp}\right) / \partial H_{\perp} \leq 0$ assures that there is not

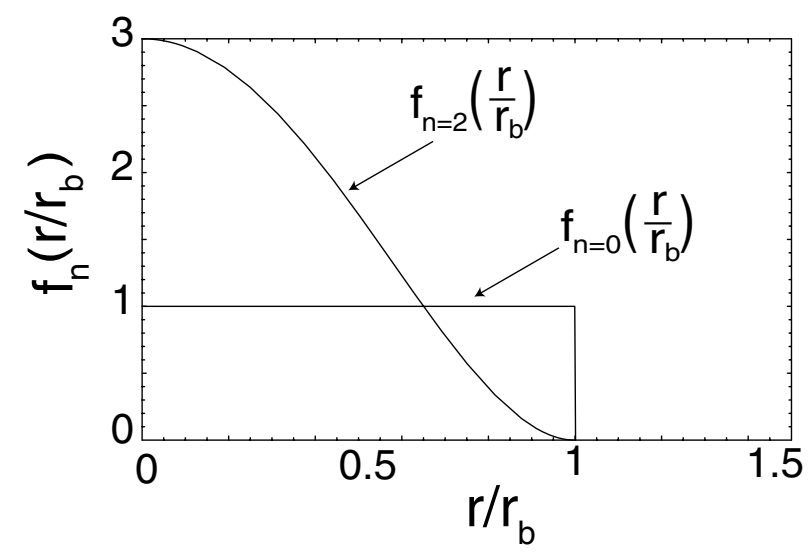

FIG. 2. Shape function profile $f_{n}\left(r / r_{b}\right)$ defined in Eq. (39) plotted versus $r / r_{b}$ for $n=0$ and $n=2$. free energy available to drive a collective transverse instability. Finally, for the class of density profiles in Eq. (34), it should be noted that the number density $n_{b}(r, z, t)$ depends on axial coordinate $z$ through the line density $\lambda_{b}(z, t)$ and the edge radius $r_{b}(z, t)$, where $r_{b}(z, t)$ depends parametrically on $\lambda_{b}(z, t)$ through Eqs. (32) and (38). We emphasize, however, that the present analysis has been carried out for a long coasting beam and has not been developed for a finite-length charge bunch. Therefore, perturbations in $\lambda_{b}(z, t)$ are envisioned to be about a constant value of line density $\lambda_{b 0}=$ const (independent of $z$ and $t$ ).

\section{Evaluation of $\left\langle E_{z}^{s}\right\rangle^{(1)}$ for fixed-shape density profile}

We now make use of Eq. (28) to evaluate the leadingorder longitudinal electric field $\left\langle E_{z}^{s}\right\rangle^{(1)}$ for the class of fixed-shape density profiles described by Eq. (34). Because the beam radius $r_{b}$ generally depends on the line density $\lambda_{b}$ [e.g., see Eq. (38)], it is evident from Eqs. (28) and (34) that the radial integrations over $n_{b} / \lambda_{b}$ required in Eq. (28) will also depend on $\lambda_{b}$. Referring to Eq. (28), it is useful to define

$$
h_{0}\left(\lambda_{b}\right)=2 \int_{0}^{r_{w}} \frac{d r}{r}\left(2 \pi \int_{0}^{r} d r r \frac{n_{b}}{\lambda_{b}}\right)^{2} .
$$

It then follows from Eqs. (28) and (41) that

$$
\left\langle E_{z}^{s}\right\rangle^{(1)}=-e_{b}\left[h_{0}\left(\lambda_{b}\right) \frac{\partial \lambda_{b}}{\partial z}+\frac{1}{2} \lambda_{b} \frac{\partial}{\partial z} h_{0}\left(\lambda_{b}\right)\right] .
$$

Equation (42) can be expressed in the compact form

$$
\left\langle E_{z}^{s}\right\rangle^{(1)}=-e_{b} g_{0} \frac{\partial \lambda_{b}}{\partial z},
$$

where the $g_{0}$ factor is defined by

$$
g_{0}\left(\lambda_{b}\right)=\frac{1}{2 \lambda_{b}} \frac{\partial}{\partial \lambda_{b}}\left[\lambda_{b}^{2} h_{0}\left(\lambda_{b}\right)\right],
$$

and $h_{0}\left(\lambda_{b}\right)$ is defined in Eq. (41).

We now evaluate $h_{0}\left(\lambda_{b}\right)$ for the class of density profiles described by Eq. (34). Substituting Eq. (34) into Eq. (41) and introducing the dimensionless radial variable $X=$ $r / r_{b}$ readily gives

$$
h_{0}\left(\lambda_{b}\right)=\ln \left(\frac{r_{w}^{2}}{r_{b}^{2}}\right)+8 \int_{0}^{1} \frac{d X}{X}\left(\int_{0}^{X} d Y Y f(Y)\right)^{2} .
$$

For specified profile shape function $f\left(r / r_{b}\right)$, note that the second term on the right-hand side of Eq. (45) is constant (independent of $\lambda_{b}$ ), whereas the logarithmic term depends on $\lambda_{b}$ through the beam radius $r_{b}\left(\lambda_{b}\right)$ [see Eq. (38)]. Substituting the profile function $f_{n}\left(r / r_{b}\right)=(n+1)(1-$ $\left.r^{2} / r_{b}^{2}\right)^{n}, n=0,1,2, \ldots$, into Eq. (45), we obtain

$$
h_{0}\left(\lambda_{b}\right)=\ln \left(\frac{r_{w}^{2}}{r_{b}^{2}}\right)+\alpha_{n},
$$


where

$$
\alpha_{n}=\sum_{m=1}^{n+1} \frac{(n+1)}{m(m+n+1)} .
$$

Note from Eq. (47) the sensitive dependence of the constant $\alpha_{n}$ on the profile shape, ranging from $\alpha_{0}=1 / 2$ for a step-function density profile $(n=0)$ to $\alpha_{1}=11 / 12$ for a parabolic profile $(n=1)$, to $\alpha_{2}=73 / 60$ for $n=2$, and so on. This, of course, affects the precise values of $h_{0}$ [Eq. (46)] and $g_{0}$ [Eq. (44)].

Finally, substituting Eq. (46) into Eq. (44), we obtain for the $g_{0}$ factor

$$
g_{0}\left(\lambda_{b}\right)=\ell n\left(\frac{r_{w}^{2}}{r_{b}^{2}}\right)+\alpha_{n}-\frac{\lambda_{b}}{2 r_{b}^{2}} \frac{\partial r_{b}^{2}}{\partial \lambda_{b}} .
$$

Substituting the expression for $r_{b}^{2}$ in Eq. (38) into Eq. (48), we readily obtain

$$
g_{0}\left(\lambda_{b}\right)=\ln \left(\frac{r_{w}^{2}}{r_{b}^{2}}\right)+\alpha_{n}-\frac{1}{2} \frac{R_{\lambda}^{2}}{\left[R_{\lambda}^{4}+4 R_{\epsilon}^{4}\right]^{1 / 2}},
$$

where $R_{\lambda}^{2}=\lambda_{b} e_{b}^{2} / m_{b} \omega_{\beta \perp}^{2}$ and $R_{\epsilon}^{2}=\tilde{\epsilon} / 2 \omega_{\beta \perp}$ are defined in Eq. (32). Equation (49) clearly displays the dependence of $g_{0}$ on the line density $\lambda_{b}$. For a low-intensity, emittance-dominated beam with $R_{\lambda}^{2} \ll 2 R_{e}^{2}$, the final term in Eq. (49) is approximately $-R_{\lambda}^{2} / 4 R_{\epsilon}^{2}$, which represents a negligibly small correction to $\ell n\left(r_{w}^{2} / r_{b}^{2}\right)+\alpha_{n}$. On the other hand, for a space-charge-dominated beam with $R_{\lambda}^{2} \gg 2 R_{\epsilon}^{2}$ the final term in Eq. (49) is approximately $-1 / 2$, representing a sizable contribution to the $g_{0}$ factor. Indeed, for the special case of a step-function density profile $\left(n=0\right.$ and $\left.\alpha_{0}=1 / 2\right)$ the second and third terms on the right-hand side of Eq. (49) exactly cancel in the limit of a space-charge-dominated beam $\left(R_{\lambda}^{2} \gg 2 R_{\epsilon}^{2}\right)$, and the $g_{0}$ factor is given approximately by $g_{0} \simeq$ $\ell n\left(r_{w}^{2} / r_{b}^{2}\right)$ as previously obtained by Reiser et al. $[52,53]$. The expression for $g_{0}\left(\lambda_{b}\right)$ in Eq. (49), of course, is valid for arbitrary beam intensity and the entire class of fixed-shape density profiles consistent with Eqs. (34) and (39) for $n=0,1,2, \ldots$.

\section{Evaluation of $\left\langle E_{z}^{s}\right\rangle^{(2)}$ for fixed-shape density profile}

We now evaluate the second-order electric field $\left\langle E_{z}^{s}\right\rangle^{(2)}$ defined in Eq. (29) for the class of fixed-shape density profiles described by Eq. (34). As a first example, we consider the case of a low-to-moderate intensity beam $\left(R_{\lambda}^{2} \ll 2 R_{\epsilon}^{2}\right)$ where the variation of beam radius $r_{b}$ with line density $\lambda_{b}$ is treated as negligibly small [see Eq. (38)]. In this case, treating $n_{b} / \lambda_{b}$ as independent of $\lambda_{b}$, Eq. (29) simplifies directly to give

$$
\left\langle E_{z}^{s}\right\rangle^{(2)}=-e_{b} g_{2} r_{w}^{2} \frac{\partial^{3} \lambda_{b}}{\partial z^{3}},
$$

where the $g_{2}$ factor occurring in Eq. (50) is defined by

$$
g_{2}=\frac{2}{r_{w}^{2}} \int_{0}^{r_{w}} \frac{d r}{r}\left(2 \pi \int_{0}^{r} d r r \frac{n_{b}}{\lambda_{b}}\right) \int_{0}^{r} d r r \int_{r}^{r_{w}} \frac{d r}{r}\left(2 \pi \int_{0}^{r} d r r \frac{n_{b}}{\lambda_{b}}\right) \text {. }
$$

We substitute Eq. (34) into Eq. (51) with shape function $f_{n}\left(r / r_{b}\right)=(n+1)\left(1-r^{2} / r_{b}^{2}\right)^{n}$ specified by Eq. (39) for $0 \leq r<r_{b}$ and $n=0,1,2, \ldots$. Some straightforward but tedious integration over $r$ in Eq. (51) gives the compact result

$$
g_{2}=\frac{1}{2}\left[1-\left(1-\beta_{n}\right) \frac{r_{b}^{2}}{r_{w}^{2}}-\frac{1}{n+2} \frac{r_{b}^{2}}{r_{w}^{2}} \ln \left(\frac{r_{w}^{2}}{r_{b}^{2}}\right)\right] .
$$

Here, the constant $\beta_{n}$ is defined by

$$
\beta_{n}=\frac{n+1}{n+2}-\sum_{m=1}^{n+1} \frac{1}{m(m+n+2)},
$$

and $r_{b}^{2}$ is related to the mean-square radius $R_{b}^{2}$ by $R_{b}^{2}=$ $r_{b}^{2} /(n+2)$ [see Eqs. (36), (38), and (40)]. It is evident from Eq. (52) that the precise value of the geometric factor $g_{2}$ exhibits a sensitive dependence on profile shape. For example, it follows from Eq. (53) that $\beta_{0}=1 / 6$ for a step-function density profile $(n=0)$, whereas $\beta_{1}=19 / 60$ for a parabolic density profile $(n=1)$. Furthermore, it follows from Eq. (52) that $g_{2}>0$, with $g_{2} \simeq 1 / 2$ for $r_{b}^{2} / r_{w}^{2} \ll 1$ and $g_{2} \simeq(1 / 2) \beta_{n}$ for $r_{b}^{2} / r_{w}^{2} \rightarrow 1$. For the special case of a step-function density profile $(n=0)$, note from Eq. (52) that the $g_{2}$ factor is given by

$$
g_{2}=\frac{1}{2}\left[1-\frac{5}{6} \frac{r_{b}^{2}}{r_{w}^{2}}-\frac{1}{2} \frac{r_{b}^{2}}{r_{w}^{2}} \ln \left(\frac{r_{w}^{2}}{r_{b}^{2}}\right)\right]
$$

Evaluation of $\left\langle E_{z}\right\rangle^{(2)}$ from Eq. (29) for arbitrary beam intensity is somewhat more complicated. For present purposes, we specialize to the case of a step-function density profile $(n=0)$. Substituting Eqs. (34) and (39) into Eq. (29), and carrying out the integrations over $r$ for $n=0$, we obtain

$$
\left\langle E_{z}^{s}\right\rangle^{(2)}=-\frac{1}{2} e_{b} r_{w}^{2}\left\{\left[1-\frac{r_{b}^{2}}{r_{w}^{2}}-\frac{1}{2} \frac{r_{b}^{2}}{r_{w}^{2}} \ln \left(\frac{r_{w}^{2}}{r_{b}^{2}}\right)\right] \frac{\partial^{3} \lambda_{b}}{\partial z^{3}}-\frac{1}{4} \ln \left(\frac{r_{w}^{2}}{r_{b}^{2}}\right) \frac{\partial^{3}}{\partial z^{3}}\left(\frac{\lambda_{b} r_{b}^{2}}{r_{w}^{2}}\right)-\frac{1}{12} \frac{r_{b}^{4}}{r_{w}^{4}} \frac{\partial^{3}}{\partial z^{3}}\left(\frac{\lambda_{b} r_{w}^{2}}{r_{b}^{2}}\right)+\frac{1}{4} \frac{r_{b}^{2}}{r_{w}^{2}} \frac{\partial^{3}}{\partial z^{3}}\left[\lambda_{b} \ln \left(\frac{r_{w}^{2}}{r_{b}^{2}}\right)+\lambda_{b}\right]\right\} .
$$

Here, for a step-function density profile, $r_{b}^{2}$ depends on $\lambda_{b}$ through the force-balance constraint $r_{b}^{2}\left(\lambda_{b}\right)=R_{\lambda}^{2}+\left(R_{\lambda}^{4}+\right.$ $\left.4 R_{\epsilon}^{4}\right)^{1 / 2}$, where $R_{\lambda}^{2}=\lambda_{b} e_{b}^{2} / m_{b} \omega_{\beta \perp}^{2}$ and $R_{\epsilon}^{2}=\tilde{\epsilon} / 2 \omega_{\beta \perp}$ [see Eqs. (32) and (38) for $n=0$ ]. For a general value of beam intensity, note from Eq. (55) that $\left\langle E_{z}^{s}\right\rangle^{(2)}$ generally has a nonlinear dependence on $\lambda_{b}$ and derivatives of $\lambda_{b}$ with respect to 
TABLE I. Values of the geometric factors $g_{0}$ and $g_{2}$ for several density profile shapes and low beam intensity $\left(R_{\lambda}^{2} \ll 2 R_{\epsilon}^{2}\right)$. Here, $\gamma=0.5772$ is Euler's constant.

\begin{tabular}{|c|c|c|c|}
\hline $\begin{array}{l}\text { Profile } \\
\text { Index } n\end{array}$ & $\begin{array}{l}\text { Normalized profile }\left(0 \leq r<r_{b}\right) \\
\frac{\pi r_{b}^{2} n_{b}}{\lambda_{b}}=(n+1)\left(1-\frac{r^{2}}{r_{b}^{2}}\right)^{n}\end{array}$ & $\begin{array}{l}\text { Geometric factor } \\
g_{0}=\ln \left(\frac{r_{w}^{2}}{r_{b}^{2}}\right)+\alpha_{n}\end{array}$ & $\begin{array}{c}\text { Geometric factor } \\
g_{2}=\frac{1}{2}\left[1-\left(1-\beta_{n}\right) \frac{r_{b}^{2}}{r_{w}^{2}}-\frac{1}{n+2} \frac{r_{b}^{2}}{r_{w}^{2}} \ln \left(\frac{r_{w}^{2}}{r_{b}^{2}}\right)\right]\end{array}$ \\
\hline 0 & 1 & $\ln \left(\frac{r_{w}^{2}}{r_{b}^{2}}\right)+\frac{1}{2}$ & $\frac{1}{2}\left[1-\frac{5}{6} \frac{r_{b}^{2}}{r_{w}^{2}}-\frac{1}{2} \frac{r_{b}^{2}}{\frac{r_{w}^{2}}{2}} \ln \left(\frac{r_{w}^{2}}{r_{b}^{2}}\right)\right]$ \\
\hline 1 & $2\left(1-\frac{r_{w}^{2}}{r_{b}^{2}}\right)$ & $\ln \left(\frac{r_{w}^{2}}{r_{b}^{2}}\right)+\frac{11}{12}$ & $\frac{1}{2}\left[1-\frac{41}{60} \frac{r_{b}^{2}}{r_{w}^{2}}-\frac{1}{3} \frac{r_{b}^{2}}{r_{w}^{2}} \ln \left(\frac{r_{w}^{2}}{r_{b}^{2}}\right)\right]$ \\
\hline 2 & $3\left(1-\frac{r_{w}^{2}}{r_{b}^{2}}\right)^{2}$ & $\ln \left(\frac{r_{w}^{2}}{r_{b}^{2}}\right)+\frac{73}{60}$ & $\frac{1}{2}\left[1-\frac{61}{105} \frac{r_{b}^{2}}{r_{w}^{2}}-\frac{1}{4} \frac{r_{b}^{2}}{r_{w}^{2}} \ln \left(\frac{r_{w}^{2}}{r_{b}^{2}}\right)\right]$ \\
\hline 3 & $4\left(1-\frac{r_{w}^{2}}{r_{b}^{2}}\right)^{3}$ & $\ln \left(\frac{r_{w}^{2}}{r_{b}^{2}}\right)+\frac{1217}{840}$ & $\frac{1}{2}\left[1-\frac{1279}{2520} \frac{r_{b}^{2}}{r_{w}^{2}}-\frac{1}{5} \frac{r_{b}^{2}}{r_{w}^{2}} \ln \left(\frac{r_{w}^{2}}{r_{b}^{2}}\right)\right]$ \\
\hline$\infty$ & $\frac{r_{b}^{2}}{R_{b}^{2}} \exp \left(-\frac{r^{2}}{R_{b}^{2}}\right)$ & $\ln \left(\frac{r_{w}^{2}}{2 R_{b}^{2}}\right)+\gamma$ & $\frac{1}{2}\left[1-(1+\gamma) \frac{R_{b}^{2}}{r_{w}^{2}}-\frac{R_{b}^{2}}{r_{w}^{2}} \ln \left(\frac{r_{w}^{2}}{2 R_{b}^{2}}\right)\right]$ \\
\hline
\end{tabular}

$z$. Indeed, Eq. (55) can generally be expressed in the form

$$
\left\langle E_{z}^{s}\right\rangle^{(2)}=-e_{b} r_{w}^{2}\left\{\tilde{g}_{2} \frac{\partial^{3} \lambda_{b}}{\partial z^{3}}+\tilde{g}_{3}\left(\frac{\partial \lambda_{b}}{\partial z}\right)^{3}+\tilde{g}_{4} \frac{\partial \lambda_{b}}{\partial z} \frac{\partial^{2} \lambda_{b}}{\partial z^{2}}\right\},
$$

where the coefficients $\tilde{g}_{2}, \tilde{g}_{3}$, and $\tilde{g}_{4}$ depend on $\lambda_{b}$, $\partial r_{b}^{2} / \partial \lambda_{b}$, etc.

In the limit of a low-intensity beam $\left(R_{\lambda}^{2} \ll 2 R_{\epsilon}^{2}\right)$, where $r_{b}^{2} \simeq 2 R_{\epsilon}^{2}=$ const (independent of $\lambda_{b}$ ), Eqs. (55) and (56) give the expected result

$$
\tilde{g}_{2}=g_{2}, \quad \tilde{g}_{3}=0, \quad \tilde{g}_{4}=0,
$$

where the geometric factor $g_{2}$ is defined in Eq. (54) for a step-function density profile. In this case, Eq. (56) reduces to $\left\langle E_{z}^{s}\right\rangle^{(2)}=-e_{b} r_{w}^{2} g_{2} \partial^{3} \lambda_{b} / \partial z^{3}$. On the other hand, for a high-intensity, space-charge dominated beam $\left(R_{\lambda}^{2} \gg 2 R_{\epsilon}^{2}\right)$, it follows that $r_{b}^{2} \simeq 2 R_{\lambda}^{2}$, and the coefficients $\tilde{g}_{2}, \tilde{g}_{3}$, and $\tilde{g}_{4}$ in Eq. (56) can be approximated by

$$
\begin{array}{ll}
\tilde{g}_{2}=\frac{1}{8}\left[4\left(1-\frac{r_{b}^{2}}{r_{w}^{2}}\right)-3 \frac{r_{b}^{2}}{r_{w}^{2}} \ln \left(\frac{r_{w}^{2}}{r_{b}^{2}}\right)\right], \quad \tilde{g}_{3}=\frac{r_{b}^{2}}{8 \lambda_{b}^{2} r_{w}^{2}}, \\
\tilde{g}_{4}=-\frac{3 r_{b}^{2}}{8 \lambda_{b} r_{w}^{2}}\left[1+2 \ln \left(\frac{r_{w}^{2}}{r_{b}^{2}}\right)\right] .
\end{array}
$$

Equations (55)-(58) clearly display the strong dependence of $\left\langle E_{z}^{s}\right\rangle^{(2)}$ on line density $\lambda_{b}$.

To conclude this section, Table I shows the values of the geometric factors $g_{0}$ and $g_{2}$ obtained from Eqs. (49) and (52) for several density profile shapes. Here, a low-intensity beam $\left(R_{\lambda}^{2} \ll 2 R_{\epsilon}^{2}\right)$ has been assumed with $r_{b}^{2} \simeq$ const (independent of $\lambda_{b}$ ).

Finally, it is important to recognize that the longitudinal dynamics considered in the present paper is assumed to be much slower than the transverse dynamics. Indeed, the longitudinal dynamics in the long-wavelength approximation considered here has characteristic frequency $\omega_{\|} \sim\left(k_{z} r_{b}\right) \omega_{p b}$ (in the beam frame), where $\omega_{p b}=\left(4 \pi n_{b} e_{b}^{2} / m_{b}\right)^{1 / 2}$ is the plasma frequency, whereas the transverse dynamics has characteristic frequency $\omega_{\perp} \sim \omega_{\beta \perp} \geq \omega_{p b}$. Since, $k_{x}^{2} r_{b}^{2} \ll 1$ is assumed [see
Eq. (23)], it therefore follows that $\omega_{\|} \ll \omega_{\perp}$. Hence, in the long-wavelength approximation we can separate the two time scales. The transverse dynamics has enough time to come to a quasiequilibrium which depends parametrically on longitudinal quantities. In this case there is a self-consistent separation of the longitudinal dynamics from the transverse beam dynamics.

\section{LONGITUDINAL VLASOV-MAXWELL EQUATIONS FOR A LOW-INTENSITY BEAM}

In Secs. II and III, we provided a systematic derivation of the longitudinal Vlasov-Maxwell equations and associated $g$ factors, valid for a wide range of beam intensity and choice of transverse density profiles. In this section, we specialize to the low-intensity regime $\left(R_{\lambda}^{2} \ll 2 R_{\epsilon}^{2}\right)$ where the dependence of the beam radius $r_{b}$ on line density $\lambda_{b}$ can be neglected. In this case, making use of Eqs. (19), (43), (49), (50), and (52), the Vlasov-Maxwell equations describing the evolution of the longitudinal beam distribution $F_{b}\left(z, p_{z}, t\right)$ and line density $\lambda_{b}(z, t)=$ $\int_{-\infty}^{\infty} d p_{z} F_{b}\left(z, p_{z}, t\right)$ can be expressed as

$$
\frac{\partial F_{b}}{\partial t}+v_{z} \frac{\partial F_{b}}{\partial z}+e_{b}\left\langle E_{z}^{s}\right\rangle \frac{\partial F_{b}}{\partial p_{z}}=0
$$

where

$$
e_{b}\left\langle E_{z}^{s}\right\rangle=-e_{b}^{2} g_{0} \frac{\partial \lambda_{b}}{\partial z}-e_{b}^{2} g_{2} r_{w}^{2} \frac{\partial^{3} \lambda_{b}}{\partial z^{3}} .
$$

Here, the geometric factors $g_{0}$ and $g_{2}$ are defined by

$$
\begin{gathered}
g_{0}=\ln \left(\frac{r_{w}^{2}}{r_{b}^{2}}\right)+\alpha_{n}, \\
g_{2}=\frac{1}{2}\left[1-\left(1-\beta_{n}\right) \frac{r_{b}^{2}}{r_{w}^{2}}-\frac{1}{n+2} \frac{r_{b}^{2}}{r_{w}^{2}} \ln \left(\frac{r_{w}^{2}}{r_{b}^{2}}\right)\right] .
\end{gathered}
$$

The constants $\alpha_{n}$ and $\beta_{n}$ are defined in Eqs. (47) and (53) for $n=0,1,2, \ldots$, and the transverse density profile assumed in deriving Eqs. (59)-(62) is specified by Eq. (43) 
with profile shape function $f_{n}\left(r / r_{b}\right)=(n+1)(1-$ $\left.r^{2} / r_{b}^{2}\right)^{n}$ for $0 \leq r<r_{b}$.

Equations (59)-(62) can be used to investigate detailed linear and nonlinear properties of the self-consistent evolution of the beam distribution function $F_{b}\left(z, p_{z}, t\right)$ and average self-generated electric field $\left\langle E_{z}^{s}\right\rangle(z, t)$ for a wide range of system parameters and choices of initial distribution function. For our purposes here, we summarize the key stability properties obtained from a linear stability analysis of Eqs. (59) and (60). Perturbations are assumed to be about a spatially uniform $(\partial / \partial z=0)$ coasting beam with equilibrium distribution function $F_{b}^{0}\left(p_{z}\right)$ and normalization $\int_{-\infty}^{\infty} d p_{z} F_{b}^{0}\left(p_{z}\right)=\lambda_{b 0}$, where $\lambda_{b 0}=$ const is the unperturbed line density. Substituting $F_{b}\left(z, p_{z}, t\right)=F_{b}^{0}\left(p_{z}\right)+\delta F_{b}\left(z, p_{z}, t\right)$ and $\lambda_{b}(z, t)=\lambda_{b 0}+$ $\delta \lambda_{b}(z, t)$ into Eqs. (59) and (60), and retaining terms linear in the perturbation amplitude, we obtain

$$
\begin{aligned}
& \left(\frac{\partial}{\partial t}+v_{z} \frac{\partial}{\partial z}\right) \delta F_{b}=-e_{b} \delta\left\langle E_{z}^{s}\right\rangle \frac{\partial}{\partial p_{z}} F_{b}^{0}\left(p_{z}\right), \\
& e_{b} \delta\left\langle E_{z}^{s}\right\rangle=-e_{b}^{2} g_{0} \frac{\partial}{\partial z} \delta \lambda_{b}-e_{b}^{2} g_{2} r_{w}^{2} \frac{\partial^{3}}{\partial z^{3}} \delta \lambda_{b},
\end{aligned}
$$

where

$$
\delta \lambda_{b}=\int_{-\infty}^{\infty} d p_{z} \delta F_{b}\left(z, p_{z}, t\right)
$$

For present purposes, the perturbations in Eqs. (63)(65) are taken to be of the form

$$
\delta \psi(z, t)=\delta \hat{\psi} \exp \left(i k_{z} z-i \omega t\right)
$$

where $k_{z}$ is the axial wave number, and $\omega$ is the complex oscillation frequency, with $\operatorname{Im} \omega>0$ corresponding to instability (temporal growth). Making use of $\partial / \partial t \rightarrow$ $-i \omega$ and $\partial / \partial z \rightarrow i k_{z}$, Eqs. (63) and (64) can be combined to give

$$
\delta F_{b}=-k_{z}\left(e_{b}^{2} g_{0}-e_{b}^{2} g_{2} k_{z}^{2} r_{w}^{2}\right) \frac{\partial F_{b}^{0} / \partial p_{z}}{\omega-k_{z} v_{z}} \delta \lambda_{b},
$$

which relates the perturbed distribution function $\delta F_{b}$ to the perturbed line density $\delta \lambda_{b}$. It is convenient to introduce effective sound speeds $u_{b 0}$ and $u_{b 2}$ associated with the geometric factors $g_{0}$ and $g_{1}$ defined by

$$
u_{b 0}^{2}=\frac{g_{0} e_{b}^{2} \lambda_{b 0}}{m_{b}}, \quad u_{b 2}^{2}=\frac{g_{2} e_{b}^{2} \lambda_{b 0}}{m_{b}} .
$$

Substituting Eqs. (67) and (68) into Eq. (65) then gives

$$
D\left(k_{z}, \omega\right) \delta \lambda_{b}=0
$$

where the dielectric function $D\left(k_{z}, \omega\right)$ is defined by

$$
D\left(k_{z}, \omega\right)=1+k_{z}\left(u_{b 0}^{2}-k_{z}^{2} r_{w}^{2} u_{b 2}^{2}\right) \frac{m_{b}}{\lambda_{b 0}} \int_{-\infty}^{\infty} d p_{z} \frac{\partial F_{b}^{0} / \partial p_{z}}{\omega-k_{z} v_{z}}
$$

for a nontrivial solution to Eq. (69) with $\delta \lambda_{b} \neq 0$; note that $D\left(k_{z}, \omega\right)=0$ plays the role of a dispersion relation that determines the complex oscillation frequency $\omega$ in terms of $k_{z}$ and properties of the equilibrium distribution function $F_{b}^{0}\left(p_{z}\right)$. Introducing the distribution function $f_{b}^{0}\left(p_{z}\right) \equiv \lambda_{b 0}^{-1} F_{b}^{0}\left(p_{z}\right)$ normalized according to $\int_{-\infty}^{\infty} d p_{z} f_{b}^{0}\left(p_{z}\right)=1$, and integrating by parts with respect to $p_{z}$ in Eq. (70) using $\partial v_{z} / \partial p_{z}=1 / m_{b}$, the dispersion relation $D\left(k_{z}, \omega\right)=0$ is readily expressed in the compact form

$D\left(k_{z}, \omega\right)=1-k_{z}^{2}\left(u_{b 0}^{2}-k_{z}^{2} r_{w}^{2} u_{b 2}^{2}\right) \int_{-\infty}^{\infty} \frac{d p_{z} f_{b}^{0}\left(p_{z}\right)}{\left(\omega-k_{z} v_{z}\right)^{2}}=0$.

The dispersion relation (71) can be used to calculate the complex oscillation frequency $\omega$ for a wide range of choices of beam distribution function $f_{b}^{0}\left(p_{z}\right)$ [1]. It is convenient to introduce the effective susceptibility $\chi\left(k_{z}, \omega\right)$ with dimensions (frequency) $)^{-2}$ defined by

$$
\chi\left(k_{z}, \omega\right)=\int_{-\infty}^{\infty} \frac{d p_{z} f_{b}^{0}\left(p_{z}\right)}{\left(\omega-k_{z} v_{z}\right)^{2}} .
$$

The resulting expressions for $\chi\left(k_{z}, \omega\right)$ are displayed in Table II for various choices of distribution function $f_{b}^{0}\left(p_{z}\right)$ ranging from a cold distribution function (entry No. 1) to a Maxwellian distribution (entry No. 5). In Table II, the constant $u_{T}$ is the effective thermal speed, which is a measure of the velocity spread of the distribution function $f_{b}^{0}\left(p_{z}\right)$. For the Maxwellian distribution in Table II (entry No. 5), the plasma dispersion function $Z(\xi)$ is defined by [1]

$$
Z(\xi)=\frac{1}{\sqrt{\pi}} \int_{-\infty}^{\infty} d u \frac{\exp \left(-u^{2}\right)}{u-\xi},
$$

where $\xi=\omega / k_{z} u_{T}$ is the normalized phase velocity. Equations (71) and (72) and Table II can be used to determine detailed wave propagation properties for several choices of beam distribution functions $f_{b}^{0}\left(p_{z}\right)$. For example, for the choice of waterbag (step-function) distribution in entry No. 2, Eqs. (71) and (72) give the dispersion relation

TABLE II. Susceptibility $\chi\left(k_{z}, \omega\right)$ [Eq. (72)] for various choices of $f_{b}^{0}\left(p_{z}\right)$.

\begin{tabular}{ccc}
\hline \hline $\begin{array}{c}\text { Entry } \\
\text { No. }\end{array}$ & Distribution & $\begin{array}{c}\text { Susceptibility } \\
f_{b}^{0}\left(p_{z}\right)\end{array}$ \\
\hline 1 & $\delta\left(p_{z}\right)$ & $\chi\left(k_{z}, \omega\right)$ [Eq. (72)] \\
2 & $f_{b}^{0}=\left\{\begin{array}{cc}\frac{1}{2 m_{b} v_{T}},\left|p_{z}\right|<m_{b} v_{T} \\
0,\left|p_{z}\right|>m_{b} v_{T} \\
\frac{m_{b} v_{T}}{\pi} \frac{1}{p_{z}^{2}+m_{b}^{2} v_{T}^{2}}\end{array}\right.$ \\
4 & $\frac{2\left(m_{b} v_{T}\right)^{2}}{\pi} \frac{1}{\left(p_{z}^{2}+m_{b}^{2} v_{T}^{2}\right)^{2}}$ & $\frac{1}{\left(\omega+k_{z}^{2} v_{T}^{2}\right)}$ \\
5 & $\frac{1}{\left(\omega+i\left|k_{z}\right| v_{T}\right)^{2}}$ \\
\hline$m_{b} v_{T}$ & $\exp \left(-\frac{p_{z}^{2}}{m_{b}^{2} v_{T}^{2}}\right)$ & $-\frac{2}{k_{z}^{2} v_{T}^{2}}\left[1+\frac{2 i\left|k_{z}\right| v_{T}}{\omega+i\left|k_{z}\right| v_{T}}\right]$ \\
\hline
\end{tabular}




$$
\omega^{2}=k_{z}^{2}\left(u_{b 0}^{2}+u_{T}^{2}-k_{z}^{2} r_{w}^{2} u_{b 2}^{2}\right) .
$$

Because $k_{z}^{2} r_{w}^{2} \ll 1$ has been assumed [Eq. (23)], the final term in Eq. (74) represents a small correction. Representing $\omega=\omega_{r}+i \omega_{i}$ where $\omega_{r}=\operatorname{Re} \omega$ and $\omega_{i}=$ $\operatorname{Im} \omega$, it is clear from Eq. (74) that the solutions are purely oscillatory with

$$
\begin{gathered}
\operatorname{Re} \omega=\omega_{r}= \pm k_{z}\left(u_{b 0}^{2}+u_{T}^{2}\right)^{1 / 2}\left(1-\frac{1}{2} k_{z}^{2} r_{w}^{2} \frac{u_{b 2}^{2}}{u_{b 0}^{2}+u_{T}^{2}}\right) \\
\operatorname{Im} \omega=\omega_{i}=0 .
\end{gathered}
$$

The solution in Eq. (75) corresponds to forward-moving $\left(\omega_{r} / k_{z}>0\right)$ and backward-moving $\left(\omega_{r} / k_{z}<0\right)$ soundlike waves propagating with constant speed $\left(u_{b 0}^{2}+u_{T}^{2}\right)^{1 / 2}$ in the beam frame, with weak cubic dispersive corrections (the term proportional to $u_{b 2}^{2} k_{z}^{3}$ ). Although the cubic dispersive corrections in Eq. (75) are small, it is precisely this effect that can lead to Korteveg-deVries-like solitons in a weakly nonlinear treatment of the nonlinear beam dynamics [56].

As a second example, we consider the Lorentzian distribution in entry No. 3 of Table II. Substituting the corresponding expression for $\chi\left(k_{z}, \omega\right)$ into Eq. (71), we obtain the dispersion relation

$$
\left(\omega+i\left|k_{z}\right| u_{T}\right)^{2}=k_{z}^{2}\left(u_{b 0}^{2}-k_{z}^{2} r_{w}^{2} u_{b 2}^{2}\right)
$$

In this case, the solutions to Eq. (76) for $k_{z}^{2} r_{w}^{2} \ll 1$ can be expressed as

$$
\begin{gathered}
\operatorname{Re} \omega=\omega_{r}= \pm k_{z} u_{b 0}\left(1-\frac{1}{2} k_{z}^{2} r_{w}^{2} \frac{u_{b 2}^{2}}{u_{b 0}^{2}}\right), \\
\operatorname{Im} \omega=\omega_{i}=-\left|k_{z}\right| u_{T} .
\end{gathered}
$$

Because $\operatorname{Im} \omega=-\left|k_{z}\right| u_{T}<0$, we note from Eq. (77) that the wave perturbation is damped due to resonant waveparticle interactions (classical Landau damping) for the choice of Lorentzian distribution function in Table II. This damping is weak $\left(\left|\omega_{i} / \omega_{r}\right| \ll 1\right)$ whenever the effective thermal speed $u_{T}$ is small in comparison with the signal speed $u_{b 0}$, and the damping is strong $\left(\left|\omega_{i} / \omega_{r}\right| \gtrsim 1\right)$ whenever $u_{T} / u_{b 0} \gtrsim 1$.

Detailed linear stability properties can, of course, be calculated for other choices of the distribution function in Table II. The main point of this section is that the longitudinal Vlasov-Maxwell equations (59) and (60) are rich in physics content, even at the linearization (small-signal) level. We defer further discussion of Eqs. (59) and (60) to a future publication in which detailed nonlinear properties are discussed.

\section{TRANSFORMED EQUATIONS IN THE LABORATORY FRAME}

The analysis in Secs. II, III, and IV was carried out in the beam frame (unprimed variables) where $\nabla \times \mathbf{E}^{s}=0$.
The transformation of the key results back to the laboratory frame (primed variables) moving with axial velocity $-V_{b}=-\beta_{b} c$ relative to the beam frame is readily accomplished according to the transformation in Eqs. (3) and (4). Without presenting algebraic details, the resulting Vlasov equation for the longitudinal distribution function $F_{b}^{\prime}\left(z^{\prime}, p_{z}^{\prime}, t^{\prime}\right)$ in the laboratory frame is given by

$$
\frac{\partial}{\partial t^{\prime}} F_{b}^{\prime}+v_{z}^{\prime} \frac{\partial}{\partial z^{\prime}} F_{b}^{\prime}+e_{b}\left\langle E_{z}^{s^{\prime}}\right\rangle^{\prime} \frac{\partial}{\partial p_{z}^{\prime}} F_{b}^{\prime}=0
$$

Here, the average longitudinal electric field $\left\langle E_{z}^{s^{\prime}}\right\rangle^{\prime}\left(z^{\prime}, t^{\prime}\right)$ in the laboratory frame is also readily expressed in terms of geometric factors $\partial \lambda_{b}^{\prime} / \partial z^{\prime}$ and higher-order derivatives of the laboratory-frame line density $\lambda_{b}^{\prime}\left(z^{\prime}, t^{\prime}\right)=$ $\int d p_{z}^{\prime} F_{b}^{\prime}\left(z^{\prime}, p_{z}^{\prime}, t^{\prime}\right)$. One key modification in the laboratory frame occurs in the radial force balance equation (30), which is replaced by

$$
\omega_{\beta \perp}^{\prime 2} R_{b}^{\prime 2}=\frac{\lambda_{b}^{\prime} e_{b}^{2}}{\gamma_{b}^{3} m_{b}}+\frac{1}{4} \frac{\tilde{\boldsymbol{\epsilon}}^{\prime 2}}{R_{b}^{\prime 2}},
$$

where $\tilde{\boldsymbol{\epsilon}}^{\prime 2} / 4 R_{b}^{\prime 2}=\left\langle v_{\perp}^{\prime 2}\right\rangle^{\prime}$. In Eq. (79), $\gamma_{b} m_{b}$ is the transverse mass, and the additional factor $1 / \gamma_{b}^{2}=1-\beta_{b}^{2}$ corresponds to a reduction in the space-charge force (the term proportional to $-\beta_{b}^{2}$ ) due to the self-magnetic field $B_{\theta}^{s^{\prime}}=-\beta_{b} E_{r}^{s^{\prime}}$ in the laboratory frame. Equation (79) readily gives [1] [compare with Eqs. (32) and (33)]

$$
R_{b}^{\prime 2}=\frac{1}{2}\left[R_{\lambda}^{\prime 2}+\left(R_{\lambda}^{\prime 4}+4 R_{\epsilon}^{\prime 4}\right)^{1 / 2}\right]
$$

where $R_{\lambda}^{\prime 2}$ and $R_{\epsilon}^{\prime 4}$ are defined by

$$
R_{\lambda}^{\prime 2}=\frac{\lambda_{b}^{\prime} e_{b}^{2}}{\gamma_{b}^{3} m_{b} \omega_{\beta \perp}^{\prime 2}}, \quad R_{\epsilon}^{\prime 4}=\frac{\tilde{\boldsymbol{\epsilon}}^{\prime 2}}{4 \omega_{\beta \perp}^{\prime 2}} .
$$

Furthermore, the edge radius $r_{b}^{\prime}\left(\lambda_{b}^{\prime}\right)$ in the laboratory frame is determined from

$$
\eta_{b} r_{b}^{\prime 2}=R_{b}^{\prime 2}
$$

for the class of fixed-shape density profiles in Eqs. (34) and (39), where $\eta_{b}=1 /(n+2)$ is defined in Eq. (40) for $n=0,1,2, \ldots$.

Finally, in the laboratory frame, some straightforward algebra shows that the formal expressions for the average longitudinal electric field $\left\langle E_{z}^{s^{\prime}}\right\rangle^{\prime}\left(z^{\prime}, t^{\prime}\right)$ in Eqs. (9), (26), and (28) are modified with the replacements $n_{b} \rightarrow n_{b}^{\prime} / \gamma_{b}$, $\lambda_{b} \rightarrow \lambda_{b}^{\prime} / \gamma_{b}$, and $\partial / \partial z \rightarrow \gamma_{b}^{-1} \partial / \partial z^{\prime}$. For example, Eq. (43) is modified to become

$$
\left\langle E_{z}^{s^{\prime}}\right\rangle^{\prime(1)}=-e_{b} g_{0}^{\prime} \frac{\partial \lambda_{b}^{\prime}}{\partial z^{\prime}},
$$

where the laboratory-frame geometric factor $g_{0}^{\prime}\left(\lambda_{b}^{\prime}\right)$ is defined by

$$
g_{0}^{\prime}=\frac{1}{\gamma_{b}^{2}} g_{0}\left(\lambda_{b}^{\prime}\right)
$$

Here, $g_{0}\left(\lambda_{b}^{\prime}\right)$ is defined in Eq. (44) [or Eq. (49)] with the 
obvious replacements $\left(r_{b}, R_{\lambda}, R_{\epsilon}, \lambda_{b}\right) \rightarrow\left(r_{b}^{\prime}, R_{\lambda}^{\prime}, R_{\epsilon}^{\prime}, \lambda_{b}^{\prime}\right)$. Similarly, in the laboratory frame, for low-to-moderate beam intensity, the second-order electric field in Eq. (50) is modified to become

$$
\left\langle E_{z}^{s^{\prime}}\right\rangle^{(2)}=-e_{b} g_{2}^{\prime} r_{w}^{2} \frac{\partial^{3} \lambda_{b}^{\prime}}{\partial z^{13}},
$$

where the geometric factor $g_{2}^{\prime}$ is defined by

$$
g_{2}^{\prime}=\frac{1}{\gamma_{b}^{4}} g_{2}
$$

and $g_{2}$ is defined in Eqs. (51)-(53). Similarly, for arbitrary beam intensity, the second-order electric field in the laboratory frame has the same form as in Eq. (56) with the right-hand side of Eq. (56) scaled by $1 / \gamma_{b}^{4}$, and $\lambda_{b}$ and $\partial / \partial z$ replaced by primed variables.

In concluding this section, it should be emphasized that the detailed theoretical analysis was relatively straightforward to carry out in the beam frame. The key results in the laboratory frame were then readily obtained by Lorentz transformation back to the laboratory frame.

\section{CONCLUSIONS}

The purpose of the present paper was to develop an improved one-dimensional kinetic model describing the self-consistent nonlinear evolution of the longitudinal distribution function and average axial electric field $\left\langle E_{z}^{s}\right\rangle(z, t)$ for a very long charge bunch (coasting beam) propagating through a cylindrical conducting pipe with radius $r_{w}$, and confined in the transverse direction by an applied focusing force $\mathbf{F}_{\text {foc }}^{\mathrm{tr}}$ described in the smooth-focusing approximation. For simplicity, to illustrate the basic approach, in the present analysis the cylindrical pipe was assumed to be perfectly conducting, and the beam transport geometry was assumed to be straight (linear). As discussed in Sec. II, the starting point was the fully nonlinear, three-dimensional Vlasov-Maxwell equations for the distribution function $f_{b}(\mathbf{x}, \mathbf{p}, t)$ and selfgenerated fields in the beam frame (unprimed variables). A reduced Vlasov equation for the longitudinal distribution function $F_{b}\left(z, p_{z}, t\right)=\int d x d y \int d p_{x} d p_{y} f_{b}(\mathbf{x}, \mathbf{p}, t)$ was obtained by integrating over the transverse phasespace variables $\left(\mathbf{x}_{\perp}, \mathbf{p}_{\perp}\right)$. Making the single ansatz that the dependence of the distribution function $f_{b}(\mathbf{x}, \mathbf{p}, t)$ on axial momentum $p_{z}$ is factorable, led to a closed system of equations describing the self-consistent evolution of the longitudinal distribution function $F_{b}\left(z, p_{z}, t\right)$ and the average axial electric field $\left\langle E_{z}^{s}\right\rangle(z, t)$. Here, assuming axisymmetry in the transverse plane $(\partial / \partial \theta=0)$, the average $\langle\cdots\rangle$ denotes the weighted transverse spatial average over $n_{b}(r, z, t) / \lambda_{b}(z, t)$ defined in Eq. (26), where $n_{b}=$ $\int d^{3} p f_{b}$ is the number density of beam particles, and $\lambda_{b}=\int d p_{z} F_{b}=\int d x d y n_{b}$ is the axial line density of beam particles. In Sec. III, we assumed that the beam dynamics was relatively quiescent in the transverse plane (no transverse instability or beam mismatch), and took the transverse density profile to have the fixed-shape form $n_{b}=\left(\lambda_{b} / \pi r_{b}^{2}\right) f\left(r / r_{b}\right)$, where the shape function $f\left(r / r_{b}\right)=(n+1)\left(1-r^{2} / r_{b}^{2}\right)^{n}$ for $0 \leq r<r_{b}$, and $f\left(r / r_{b}\right)=0$ for $r_{b}<r \leq r_{w}$. Here, $n=0,1,2, \ldots$ is an integer, with $n=0$ corresponding to a step-function density profile, and $r_{b}$ is the edge radius of the beam. Moreover, the root-mean-square beam radius $R_{b}=$ $\left\langle r^{2}\right\rangle^{1 / 2}$ and edge radius $r_{b}$ generally depend on the line density through the radial force-balance condition in Eqs. (33) and (38), respectively. Of course, this dependence is weak ( $r_{b} \simeq$ const) for a low-intensity, emittancedominated beam, whereas $r_{b}^{2} \propto \lambda_{b}$ for a very-low-emittance, space-charge-dominated beam. Denoting $\partial / \partial z \sim$ $L_{z}^{-1} \sim k_{z}$, we assumed slow axial variations of $F_{b}\left(z, p_{z}, t\right)$ and $\lambda_{b}(z, t)$ with $k_{z}^{2} r_{w}^{2} \ll 1$. The average electric field $\left\langle E_{z}^{s}\right\rangle(z, t)$, expressed in terms of $\partial \lambda_{b} / \partial z$ and higher-order derivatives, together with closed forms for the corresponding geometric factors were then calculated selfconsistently in Sec. IV correct to $0\left(k_{z}^{2} r_{w}^{2}\right)$ for the class of bell-shaped density profiles with $n=0,1,2, \ldots$, described above. The results showed a strong dependence of the geometric factors on profile shape and beam intensity $\lambda_{b}$.

In Sec. IV, as a simple application, the resulting coupled equations for the longitudinal distribution function $f_{b}\left(z, p_{z}, t\right)$ and the average electric field $\left\langle E_{z}^{s}\right\rangle(z, t)$ were solved in the linearization approximation for the case of low-to-moderate beam intensity treating $r_{b} \simeq$ const (independent of $\lambda_{b}$ ). As expected, the analysis led to collective oscillations with sound-wave-like characteristics modified by cubic dispersion. The detailed oscillation and damping properties of the perturbation, of course, depended on the choice of equilibrium distribution function $F_{b}^{0}\left(p_{z}\right)$ about which the system is perturbed. Finally, in Sec. V, the key results derived in the beam frame were transformed back to the laboratory frame to facilitate practical applications of the kinetic model of the longitudinal beam dynamics developed here.

In conclusion, the present analysis has developed an improved one-dimensional kinetic model describing the nonlinear evolution of the longitudinal distribution function $F_{b}\left(z, p_{z}, t\right)$ and average electric field $\left\langle E_{z}^{s}\right\rangle(z, t)$. The analysis has been carried out for arbitrary beam intensity and general density profile shape, leading to important generalizations of previous $g$-factor models [see Eqs. (43)-(45), (50), and (51)]. Since the longitudinal beam dynamics typically depends in detail on the precise values and degree of nonlinearity of the $g$ factors, it is expected that the new results presented here will have several key applications.

In future investigations, the kinetic model for the longitudinal beam dynamics developed here will be applied to various investigations, including the nonlinear Korteweg-deVries-like evolution of axial disturbances and the self-consistent generation of compressional solitons [56]. 


\section{ACKNOWLEDGMENTS}

This research was supported by the U.S. Department of Energy.

[1] R. C. Davidson and H. Qin, Physics of Intense Charged Particle Beams in High Energy Accelerators (World Scientific, Singapore, 2001), and references therein.

[2] M. Reiser, Theory and Design of Charged Particle Beams (Wiley, New York, 1994).

[3] J. D. Lawson, The Physics of Charged-Particle Beams (Oxford Science Publications, New York, 1988).

[4] A.W. Chao, Physics of Collective Beam Instabilities in High Energy Accelerators (Wiley, New York, 1993).

[5] D. A. Edwards and M. J. Syphers, An Introduction to the Physics of High-Energy Accelerators (Wiley, New York, 1993).

[6] T.P. Wangler, Principles of $R F$ Linear Accelerators (Wiley, New York, 1998).

[7] See, for example, Proceedings of the 2001 Particle Accelerator Conference (IEEE Catalog No. 01CH37268, 2001), pp. 1-4098.

[8] See, for example, Proceedings of the 1999 International Heavy Ion Fusion Symposium [Nucl. Instrum. Methods Phys. Res., Sect. A 464, 1 (2001)].

[9] I. M. Kapchinskij and V.V. Vladimirskij, in Proceedings of the International Conference on High Energy Accelerators and Instrumentation (CERN Scientific Information Service, Geneva, 1959), p. 274.

[10] R. L. Gluckstern, in Proceedings of the 1970 Proton Linear Accelerator Conference, Batavia, IL, edited by M. R. Tracy (National Accelerator Laboratory, Batavia, IL, 1971), p. 811.

[11] T.-S. Wang and I. Smith, Part. Accel. 12, 247 (1982).

[12] J. Hofmann, L. J. Laslett, L. Smith, and I. Haber, Part. Accel. 13, 145 (1983).

[13] J. Struckmeier, J. Klabunde, and M. Reiser, Part. Accel. 15, 47 (1984).

[14] I. Hofmann and J. Struckmeier, Part. Accel. 21, 69 (1987).

[15] J. Struckmeier and I. Hofmann, Part. Accel. 39, 219 (1992).

[16] R. L. Gluckstern, W.-H. Cheng, and H. Ye, Phys. Rev. Lett. 75, 2835 (1995).

[17] N. Brown and M. Reiser, Phys. Plasmas 2, 965 (1995).

[18] C. Chen and R.C. Davidson, Phys. Rev. E 49, 5679 (1994).

[19] C. Chen, R. Pakter, and R. C. Davidson, Phys. Rev. Lett. 79, 225 (1997).

[20] R. C. Davidson and C. Chen, Part. Accel. 59, 175 (1998).

[21] R. C. Davidson, W.W. Lee, and P. Stoltz, Phys. Plasmas 5, 279 (1998).

[22] R. C. Davidson, Phys. Rev. Lett. 81, 991 (1998).

[23] R. C. Davidson, Phys. Plasmas 5, 3459 (1998).

[24] R. C. Davidson and H. Qin, Phys. Rev. ST Accel. Beams 2, 114401 (1999).
[25] P. Stoltz, R. C. Davidson, and W.W. Lee, Phys. Plasmas 6, 298 (1999).

[26] H. Qin, R. C. Davidson, and W.W. Lee, Phys. Rev. ST Accel. Beams 3, 084401 (2000); 3, 109901 (2000).

[27] Q. Qian, W.W. Lee, and R. C. Davidson, Phys. Plasmas 4, 1915 (1997).

[28] A. Friedman, D. P. Grote, and I. Haber, Phys. Fluids B 4, 2203 (1992).

[29] R. C. Davidson, H. Qin, P. Stoltz, and T.-S. Wang, Phys. Rev. ST Accel. Beams 2, 054401 (1999).

[30] J. Haber, A. Friedman, D. P. Grote, S. M. Lund, and R. A. Kishek, Phys. Plasmas 6, 2254 (1999).

[31] R. A. Kishek, P. G. O'Shea, and M. Reiser, Phys. Rev. Lett. 85, 4514 (2000).

[32] R. C. Davidson, H. Qin, and P. J. Channell, Phys. Rev. ST Accel. Beams 2, 074401 (1999); 3, 029901 (2000).

[33] P. J. Channell, Phys. Plasmas 6, 982 (1999).

[34] H. S. Uhm and R.C. Davidson, Phys. Rev. ST Accel. Beams 6, 034204 (2003).

[35] H.S. Uhm, R.C. Davidson, and I. Kaganovich, Phys. Plasmas 8, 4637 (2001).

[36] R. C. Davidson and H. Qin, Phys. Rev. ST Accel. Beams 4, 104401 (2001).

[37] E. A. Startsev, R. C. Davidson, and H. Qin, Phys. Plasmas 9, 3138 (2002).

[38] E. A. Startsev and R. C. Davidson, Phys. Rev. ST Accel. Beams 6, 044401 (2003).

[39] S. I. Tzenov and R. C. Davidson, Phys. Rev. ST Accel. Beams 5, 021001 (2002).

[40] I. Hofmann, Z. Naturforsch. 37A, 939 (1982).

[41] I. Hofmann, Laser Part. Beams 3, 1 (1985).

[42] O. Boine-Frankenheim, I. Hofmann, and G. Rumulo, Phys. Rev. Lett. 82, 3256 (1999).

[43] L. K. Spentzouris, J.-F. Ostiguy, and P. L. Colestock, Phys. Rev. Lett. 76, 620 (1996).

[44] L. K. Spentzouris, P. L. Colestock, and C. Bhat, in Proceedings of the 1999 Particle Accelerator Conference, New York (IEEE, Piscataway, NJ, 1999), Vol. 1, p. 114.

[45] R. Fedele, G. Miele, L. Palumbo, and V. G. Vaccaro, Phys. Lett. A 179, 407 (1993).

[46] H. Schamel, Phys. Rev. Lett. 79, 2811 (1997).

[47] H. Schamel and R. Fedele, Phys. Plasmas 7, 3421 (2000).

[48] A. Hofmann, CERN Report No. 77-13, 1977.

[49] D. Neuffer, Part. Accel. 11, 23 (1980).

[50] C. K. Allen, N. Brown, and M. Reiser, Part. Accel. 45, 149 (1994).

[51] W. M. Sharp, A. Friedman, and D. P. Grote, Fusion Eng. Des. 32, 201 (1996).

[52] J. G. Wang, H. Suk, and M. Reiser, Phys. Rev. Lett. 72, 2029 (1994).

[53] See, for example, p. 505 of Ref. [2].

[54] S. Koscielniak, in Proceedings of the 2001 Particle Accelerator Conference (IEEE O-7803-7191-2001, 2001), p. 2970.

[55] S. Koscielniak, S. Hancock, and M. Lindroos, Phys. Rev. ST Accel. Beams 4, 044201 (2001).

[56] R. C. Davidson (to be published). 